\title{
General entropy-like uncertainty relations in finite dimensions
}

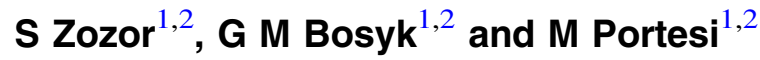 \\ ${ }^{1}$ Laboratoire Grenoblois d'Image, Parole, Signal et Automatique (GIPSA-Lab, CNRS), \\ 11 rue des Mathématiques, F-38402 Saint Martin d'Hères, France \\ ${ }^{2}$ Instituto de Física La Plata (IFLP), CONICET, and Departamento de Física, Facultad \\ de Ciencias Exactas, Universidad Nacional de La Plata, C.C. 67, 1900 La Plata, \\ Argentina
}

E-mail: steeve.zozor@gipsa-lab.inpg.fr, gbosyk@ fisica.unlp.edu.ar and portesi@fisica.unlp.edu.ar

Received 28 February 2014, revised 14 August 2014

Accepted for publication 2 October 2014

Published 20 November 2014

\begin{abstract}
We revisit entropic formulations of the uncertainty principle (UP) for an arbitrary pair of positive operator-valued measures (POVM) $A$ and $B$, acting on finite dimensional Hilbert space. Salicrú generalized $(h, \phi)$-entropies, including Rényi and Tsallis ones among others, are used as uncertainty measures associated with the distribution probabilities corresponding to the outcomes of the observables. We obtain a nontrivial lower bound for the sum of generalized entropies for any pair of entropic functionals, which is valid for both pure and mixed states. The bound depends on the overlap triplet $\left(c_{A}, c_{B}, c_{A, B}\right)$ with $c_{A}$ (respectively $c_{B}$ ) being the overlap between the elements of the POVM $A$ (respectively $B$ ) and $c_{A, B}$ the overlap between the pair of POVM. Our approach is inspired by that of de Vicente and Sánchez-Ruiz (2008 Phys. Rev. A 77 042110) and consists in a minimization of the entropy sum subject to the Landau-Pollak inequality that links the maximum probabilities of both observables. We solve the constrained optimization problem in a geometrical way and furthermore, when dealing with Rényi or Tsallis entropic formulations of the UP, we overcome the Hölder conjugacy constraint imposed on the entropic indices by the Riesz-Thorin theorem. In the case of nondegenerate observables, we show that for given $c_{A, B}>\frac{1}{\sqrt{2}}$, the bound obtained is optimal; and that, for Rényi entropies, our bound improves Deutsch one, but Maassen-Uffink bound prevails when $c_{A, B} \leqslant \frac{1}{2}$. Finally, we illustrate by comparing our bound with known previous results in particular cases of Rényi and Tsallis entropies.
\end{abstract}


Keywords: entropic uncertainty relation, generalized Salicrú et al $(h, \phi)$ entropies, pure and mixed qudit states

PACS numbers: 03.65.Ta, 89.70.Cf, 03.65.Ca, 03.65.Aa

\section{Introduction}

The uncertainty principle (UP), originally formulated by Heisenberg [1], is one the most characteristic features of the quantum world. The principle establishes that one cannot predict with certainty and simultaneously the outcomes of two (or more) incompatible measurements. The study of quantitative formulations of this principle has a long outstanding history. First formulations made use of variances as uncertainty measures and the principle was described state by state by the existence of a lower bound for the product of the variances [1-3]. However, such formulations are not always adequate since the variance is not always convenient for describing the uncertainty of a random variable. For instance, there exist variables with infinite variance [4]. Moreover, in the case of discrete-spectrum observables, the universal (state-independent) lower bound becomes trivial (zero), and thus Heisenberg-like inequalities do not quantify the UP [5-9]. For these reasons, many authors attempted and still attempt to propose alternative formulations, using other uncertainty measures. One possibility consists in using information-theoretic measures [10-12], leading to entropic uncertainty relations (EURs). In this line, pioneering works by Hirschman [13], Bialynicki-Birula and Mycielski [14] based on important results due to Beckner [15], Deutsch [5], or Maassen and Uffink (MU) [6] who proved a result conjectured by Kraus [16], have given rise to different formulations of the principle based on Shannon and generalized one-parameter information entropies, or on entropic moments [17-45]. Versions using the sum of variances (instead of their product) [46], the Fisher information [47-49], or moments of various orders [50] have also been developed.

In this contribution, we focus on the formulation of the UP in the case of finite dimensions by using $(h, \phi)$-entropies, a generalization of the Shannon entropy due to Salicrú et al [51, 52]. In particular, we deal with two well-known one-parameter entropy families, the Rényi and Tsallis ones. Our aim is to obtain a universal and nontrivial bound for the sum of the entropies associated with the outcomes of a pair of positive operator-valued measures (POVM). In order to do this, we follow a method similar to that of de Vicente and SánchezRuiz in [26], solving the minimization problem for the sum of generalized entropies subject to the Landau-Pollak inequality [53]. We develop a geometrical approach to the problem.

The paper is organized as follows. In section 2, we begin with basic definitions and notation, we present the problem, and we summarize previous results on EURs that deal with Rényi or Tsallis entropies. In section 3, we give our main results concerning general entropylike formulations of the UP in finite dimensions. For the sake of comparison with existing bounds in the literature, in section 4 we choose some particular cases. A discussion is provided in section 5. The proofs of our results are given in detail in a series of appendices.

\section{Statement of the problem: notation and previous results}

\subsection{Generalized entropies}

We are interested in quantitative formulations of the UP, particularly through the use of information-theoretic quantities. More precisely, as measure of ignorance or of lack of information we employ Salicrú et al $(h, \phi)$-entropies [51, 52] 


$$
H_{(h, \phi)}(p)=h\left(\sum_{k=1}^{N} \phi\left(p_{k}\right)\right)
$$

for any probability vector $p \in \mathcal{P}_{N}$ (the set of probability vectors in $\mathbb{R}_{+}^{N}$ ) and where the entropic functionals $\phi:[0 ; 1] \mapsto \mathbb{R}$ and $h: \mathbb{R} \mapsto \mathbb{R}$ are such that, either $\phi$ is concave and $h$ is increasing, or $\phi$ is convex and $h$ is decreasing. We restrict here to employ entropic functionals such that

- $\phi$ is continuous and strictly concave or strictly convex,

- $h$ is continuous and strictly monotone,

- $\phi(0)=0$ (so that the 'elementary' uncertainty associated to a event with zero-probability is zero),

- $h(\phi(1))=0$ (without loss of generality).

Many of the well-known cases in the literature satisfy these assumptions (see [51, 52] for a list of examples). Among them, the most renowned ones are

- Shannon entropy [10], given by $\phi(x)=-x \log x$ and $h(x)=x$, where $\log$ stands for the natural logarithm, corresponding to

$$
H(p)=-\sum_{k} p_{k} \log p_{k}
$$

- Rényi entropies [11], introduced in the domain of mathematics from the same axiomatics as Shannon but relaxing only one property (recursivity is generalized); it is given by $\phi(x)=x^{\lambda}$, and $h(x)=\frac{\log x}{1-\lambda}$, where $\lambda \geqslant 0$ is the entropic index,

$$
R_{\lambda}(p)=\frac{1}{1-\lambda} \log \left(\sum_{k} p_{k}^{\lambda}\right) .
$$

- Tsallis entropies, firstly introduced by Havrda and Charvát [54] from an axiomatics quite close to that of Shannon, then by Daróczy [55] through a generalization of a functional equation satisfied by the Shannon entropy, and finally by Tsallis [56] in the domain of nonextensive physics; it is given by $\phi(x)=x^{\lambda}, \lambda \geqslant 0$, and $h(x)=\frac{x-1}{1-\lambda}$,

$$
S_{\lambda}(p)=\frac{1-\sum_{k} p_{k}^{\lambda}}{\lambda-1} .
$$

The last two cases belong to a general one-parameter family given by $\phi(x)=x^{\lambda}$ and $h(x)=\frac{f(x)}{1-\lambda}$,

$$
F_{\lambda}(p)=\frac{f\left(\sum_{k} p_{k}^{\lambda}\right)}{1-\lambda}
$$

with $f$ increasing and $f(1)=0$, and where the entropic index $\lambda$ plays the role of a 'magnifying glass', in the following sense: when $\lambda<1$, the contribution of the different terms in the sum $\sum_{k} p_{k}^{\lambda}$ becomes more uniform with respect to the case $\lambda=1$, thus stressing the tails of the distribution; conversely, when $\lambda>1$, the leading probabilities of the distribution 
are stressed in the summation. As an extreme example, for $\lambda=0$ the generalized entropy $F_{0}(p)$ is simply a function of the number of nonzero components of the probability vector $p$, regardless of the values of these probabilities; this measure is closely linked to the $l^{0}$ quasinorm which measures the sparsity of a representation in signal processing [57-59]. If additionally $f$ is differentiable, with $f^{\prime}(1)=1$, the Shannon entropy is recovered from $F_{\lambda}$ entropies when $\lambda \rightarrow 1$.

The generalized $(h, \phi)$-entropies (1) satisfy usual properties as:

- $H_{(h, \phi)}(p)$ is a Schur-concave function of its argument, that is, if $p$ is majorized ${ }^{3}$ by $q$, which is denoted $p \prec q$, then $H_{(h, \phi)}(p) \geqslant H_{(h, \phi)}(q)$. This property is a consequence of Karamata inequality that states that if $\phi$ is convex (respectively concave), then $p \mapsto \sum_{k} \phi\left(p_{k}\right)$ is Schur-convex (respectively Schur-concave) (see [60] or [61, chapter 3, proposition C.1]), together with the decreasing (respectively increasing) property of $h$. The property of Schur-concavity is useful in some problems of combinatorial, numerical or statistical analysis [61].

- $H_{(h, \phi)}(p) \geqslant 0 \forall p \in \mathcal{P}_{N}$, with equality iff the probability distribution is a Kronecker delta: $p_{k}=\delta_{k, i}$ for certain $i$, that is, the $i$ th-outcome appears with certainty so that the ignorance is zero. This property is a consequence of Schur-concavity of $H_{(h, \phi)}$ since $p \prec\left[\begin{array}{llll}1 & 0 & \cdots & 0\end{array}\right]^{t}$, together with $h(\phi(1))=0$.

- $H_{(h, \phi)}(p) \leqslant h\left(N \phi\left(\frac{1}{N}\right)\right) \forall p \in \mathcal{P}_{N}$, with equality iff the probability distribution is uniform: $p_{k}=\frac{1}{N}$ for all $k$, that is, all outcomes appear with equal probability so that the uncertainty is maximal. Again, this property is a consequence of Schur-concavity of $H_{(h, \phi)}$ since $\left[\begin{array}{lll}\frac{1}{N} & \cdots & \frac{1}{N}\end{array}\right]^{t} \prec p$ (see [61, equation (8) p 9]).

- $H_{(h, \phi)}(p)$ is a concave function of $p$ if $h$ is concave; this is due to the facts that: (i) for concave (respectively convex) function $\phi$, function $p \mapsto \sum_{k} \phi\left(p_{k}\right)$ is concave (respectively convex) [62], and (ii) function $h$ is increasing (respectively decreasing). This property is useful in optimization problems $[62,63]$. Shannon entropy is known to be concave [12]. Rényi entropy is concave for $\lambda \in[0 ; 1]$; and in fact, it can be shown that there exists an $N$-dependent index $\lambda_{*}(N)$ greater than 1 , up to which Rényi entropy remains concave [64, p. 57]. Tsallis entropy is concave for any index $\lambda \geqslant 0$.

Furthermore, the one-parameter entropy $F_{\lambda}$ is a decreasing function in terms of $\lambda$ for fixed $p$. With the positivity of $f$, this ensures the convergence of $F_{\lambda}$ (at least simply) when $\lambda \rightarrow+\infty$ so that $F_{\infty}$ could be called minimal generalized $F_{\lambda}$-entropy (when the limit is not identically zero).

Finally, note that from the strict monotony of the function $h$, there exists a one-to-one mapping between two generalized entropies sharing the same functional $\phi$, say $(h, \phi)$ and $(g, \phi)$, under the form $H_{(h, \phi)}(p)=h\left(g^{-1}\left(H_{(g, \phi)}(p)\right)\right)$. For instance, the one-to-one mappings between Rényi entropy (3) and Tsallis entropy (4), for a given $\lambda$, are

$$
S_{\lambda}(p)=\frac{1-\exp \left((1-\lambda) R_{\lambda}(p)\right)}{\lambda-1}
$$

\footnotetext{
3 By definition, $p<q$ means that, $\sum_{k=1}^{m} p_{k}^{\downarrow} \leqslant \sum_{k=1}^{m} q_{k}^{\downarrow}, m=1, \ldots, N-1$, and $\sum_{k=1}^{N} p_{k}=\sum_{k=1}^{N} q_{k}$, where.$\downarrow$ means that the components are rearranged in decreasing order.
} 
and

$$
R_{\lambda}(p)=\frac{1}{1-\lambda} \log \left(1+(1-\lambda) S_{\lambda}(p)\right)
$$

\subsection{Entropic uncertainty relations}

Let $\mathcal{H}$ be an $N$-dimensional Hilbert space. A general quantum measurement is described by a set of positive operator-valued measures (POVM). This is a set $A=\left\{A_{i}\right\}_{i=1}^{N_{A}}$ of Hermitian positive semidefinite operators satisfying the completeness relation $\sum_{i=1}^{N_{A}} A_{i}=I$, where $I$ is the identity operator and $N_{A}$ is the number of outcomes. For given POVM $A$ and quantum system described by a density operator $\rho$ (Hermitian, positive semidefinite with unit trace) acting on $\mathcal{H}$, the probability of the $i$ th outcome is equal to $p_{i}(A, \rho)=\operatorname{Tr}\left(A_{i} \rho\right)$.

In this contribution, we consider the $(h, \phi)$-entropies (1) for the probability vectors

$$
\begin{aligned}
& p(A, \rho)=\left[p_{1}(A, \rho) \cdots p_{N_{A}}(A, \rho)\right]^{t} \text { with } \quad p_{i}(A, \rho)=\operatorname{Tr}\left(A_{i} \rho\right) \quad \text { and } \\
& p(B, \rho)=\left[p_{1}(A, \rho) \cdots p_{N_{B}}(B, \rho)\right]^{t} \text { with } \quad p_{j}(B, \rho)=\operatorname{Tr}\left(B_{j} \rho\right)
\end{aligned}
$$

associated with the measurements of two POVM sets $A$ and $B$, respectively.

The fact that the sum of $(h, \phi)$-entropies is lower bounded gives rise to an entropy-like formulation of the UP, that is, inequalities of the form

$$
H_{\left(h_{A}, \phi_{A}\right)}(p(A, \rho))+H_{\left(h_{B}, \phi_{B}\right)}(p(B, \rho)) \geqslant \mathcal{B}_{\left(h_{A}, \phi_{A}\right),\left(h_{B}, \phi_{B}\right)}
$$

for any two pairs $\left(h_{A}, \phi_{A}\right)$ and $\left(h_{B}, \phi_{B}\right)$ of entropic functionals, where the bound $\mathcal{B}_{\left(h_{A}, \phi_{A}\right),\left(h_{B}, \phi_{B}\right)}$ is nontrivial, i.e., nonzero, and universal in the sense of being independent of the state $\rho$ of the quantum system. In particular, dealing with the family $F_{\lambda}$, we focus on the case, where $f$ is the same for both entropies, but with an arbitrary pair $(\alpha, \beta)$ of nonnegative entropic indices. The ultimate goal is to find the optimal bound, which by definition is obtained by minimization of the left-hand side, i.e.

$$
\overline{\mathcal{B}}_{\left(h_{A}, \phi_{A}\right),\left(h_{B}, \phi_{B}\right)}(A, B) \equiv \min _{\rho}\left\{H_{\left(h_{A}, \phi_{A}\right)}(p(A, \rho))+H_{\left(h_{B}, \phi_{B}\right)}(p(B, \rho))\right\} .
$$

In the case of two nondegenerate quantum measurements, the optimal bound depends on the transformation matrix $T$ whose entries are given by

$$
T_{i j}=\left\langle b_{j} \mid a_{i}\right\rangle \text {, }
$$

where $\left\{\left|a_{i}\right\rangle\right\}_{i=1}^{N}$ and $\left\{\left|b_{j}\right\rangle\right\}_{j=1}^{N}$ are eigenbases of $A$ and $B$, respectively $\left(A_{i}=\left|a_{i}\right\rangle\left\langle a_{i}\right|\right.$, $\left.B_{j}=\left|b_{j}\right\rangle\left\langle b_{j}\right|, N_{A}=N_{B}=N\right)$. From the orthonormality of the bases, $T \in \mathcal{V}(N)$, where $\mathcal{V}(N)$ denotes the set of $N \times N$ unitary matrices. A relevant characteristic of such a unitary matrix is its greatest-modulus element

$$
c(T)=\max _{i, j}\left|\left\langle b_{j} \mid a_{i}\right\rangle\right|
$$

the so-called overlap between the eigenbases of $A$ and $B$. From the unitary property of matrix $T$, the overlap is in the range $c \in\left[\frac{1}{\sqrt{N}} ; 1\right]$. The case $c=\frac{1}{\sqrt{N}}$ corresponds to $A$ and $B$ being complementary observables, meaning that maximum certainty in the measure of one of them, implies maximum ignorance about the other. In the opposite extreme case, $c=1$ corresponds to observables $A$ and $B$ sharing (at least) an eigenvector; this situation happens for example when the observables commute. 
In this nondegenerate context, to find the optimal bound depending on the transformation matrix is a difficult problem in general; a weaker problem is to restrict to bounds depending on the overlap $c$ instead of on the whole matrix $T$. Thus, the optimal $c$-dependent bound writes

$$
\widetilde{\mathcal{B}}_{\left(h_{A}, \phi_{A}\right),\left(h_{B}, \phi_{B}\right) ; N}(c)=\min _{T \in \mathcal{V}(N): c(T)=c} \overline{\mathcal{B}}_{\left(h_{A}, \phi_{A}\right),\left(h_{B}, \phi_{B}\right)}(T) .
$$

We call $\widetilde{\mathcal{B}}_{\left(h_{A}, \phi_{A}\right),\left(h_{B}, \phi_{B}\right) ; N}(c)$ the c-optimal bound in order to distinguish it from $\overline{\mathcal{B}}_{\left(h_{A}, \phi_{A}\right),\left(h_{B}, \phi_{B}\right)}(T)$ that we call T-optimal bound.

Similarly, in the general POVM framework, finding the $(A, B)$-optimal bound equation (9) is a difficult task. In this context, a relevant characteristic of the pair $(A, B)$ is the triplet of overlaps

$$
\boldsymbol{c}(A, B)=\left(c_{A}, c_{B}, c_{A, B}\right) \quad \text { where }\left\{\begin{array}{l}
c_{A}=\max _{i}\left\|\sqrt{A_{i}}\right\| \\
c_{B}=\max _{j}\left\|\sqrt{B_{j}}\right\| \\
c_{A, B}=\max _{i, j}\left\|\sqrt{A_{i}} \sqrt{B_{j}}\right\|
\end{array}\right.
$$

(in the nondegenerate case, $c=(1,1, c)$ ). A weaker problem is again to restrict to bounds depending only on $\boldsymbol{c}$, the $\boldsymbol{c}$-optimal bound being

$$
\widetilde{\mathcal{B}}_{\left(h_{A}, \phi_{A}\right),\left(h_{B}, \phi_{B}\right) ; N}(\boldsymbol{c})=\min _{(A, B): c(A, B)=c} \overline{\mathcal{B}}_{\left(h_{A}, \phi_{A}\right),\left(h_{B}, \phi_{B}\right)}(A, B)
$$

with $N=\left(N_{A}, N_{B}, N\right)$.

The study of entropic formulations to quantify the UP is not new and has been addressed in various contexts $[5,6,16-45]$. However, the problem of finding $c$-optimal (respectively $c$ optimal) or $(A, B)$-optimal (respectively $T$-optimal) bounds in the form posed in equations (8)-(14) still remains open in many cases. Moreover, many available results correspond to Rényi or Tsallis entropies with conjugated indices (in the sense of Hölder: $\frac{1}{2 \alpha}+\frac{1}{2 \beta}=1$ ) as they are based on the Riesz-Thorin theorem [65]; however, recently some results were derived for nonconjugated indices in some particular situations.

For the sake of later comparison we summarize existing bounds, dealing in particular with Rényi or Tsallis entropies, classified by the entropic measure used and the entropic indices involved. To fix notation, we define the following regions in the $\alpha-\beta$-plane:

$$
\left\{\begin{array}{l}
\mathcal{C}=\left\{(\alpha, \beta) \in\left(\frac{1}{2} ;+\infty\right)^{2}: \beta=\frac{\alpha}{2 \alpha-1}\right\} \\
\underline{\mathcal{C}}=\left[0 ; \frac{1}{2}\right] \times \mathbb{R}_{+} \bigcup\left\{(\alpha, \beta) \in \mathbb{R}_{+}^{2}: \alpha>\frac{1}{2}, \beta<\frac{\alpha}{2 \alpha-1}\right\} \\
\overline{\mathcal{C}}=\left\{(\alpha, \beta) \in \mathbb{R}_{+}^{2}: \alpha>\frac{1}{2}, \beta>\frac{\alpha}{2 \alpha-1}\right\}
\end{array}\right.
$$

which are called conjugacy curve and regions 'below' and 'above' the conjugacy curve, respectively (see figure 1).

Results available in the literature comprise the following:

- Shannon entropy: $(\alpha, \beta)=(1,1)$

$\diamond$ Deutsch obtained the first bound in 1983 [5], which is given by $\mathcal{B}^{D}(c)=-2 \log \left(\frac{1+c}{2}\right)$ 


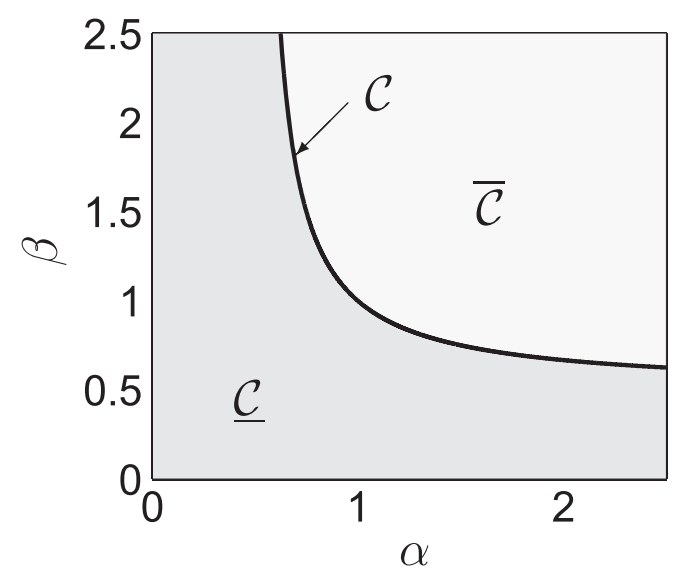

Figure 1. The conjugacy curve $\mathcal{C}$ is represented by the solid line (the positive branch of the hyperbola $\frac{1}{2 \alpha}+\frac{1}{2 \beta}=1$ ), while the region $\underline{\mathcal{C}}$ 'below' this curve is in dark gray, and the region $\bar{C}$ 'above' that curve is represented in light gray.

$\diamond$ MU improved Deutsch bound by using the Riesz-Thorin theorem, in the context of pure states. Their bound is $\mathcal{B}^{\mathrm{MU}}(c)=-2 \log c$ and it is not optimal, except for complementary observables, that is, for $c=\frac{1}{\sqrt{N}}$.

$\diamond$ de Vicente and Sanchez-Ruiz [26, 34] improved MU bound in the range $c \in\left[c^{*} ; 1\right]$ with $c^{*} \simeq 0.834$ by using the Landau-Pollak inequality that links $\max _{i} p_{i}(A, \rho)$ and $\max _{j} p_{j}(B, \rho)$, in the context of pure states. This bound is not optimal, except for complementary observables (see also [23, 27]) or for qubits $(N=2)[22,40]$.

$\diamond$ Recently, Coles and Piani (CP) [44] improved the MU bound in the whole range of the overlap $c$, indeed they obtained the bound $\mathcal{B}^{\mathrm{CP}}\left(c, c_{2}\right)=-2 \log c+(1-c) \log \frac{c}{c_{2}}$, where $c_{2}$ is the second largest value among the $\left|T_{i j}\right|$. Moreover, the authors obtained a stronger but implicit bound $\mathcal{B}^{\overline{\mathrm{CP}}}(T)$ and generalized their results for POVMs and bipartite scenarios (see also [45]).

- Rényi entropies:

$\diamond$ For $(\alpha, \beta) \in \mathcal{C}$, the MU bound $\mathcal{B}^{\mathrm{MU}}(c)$ remains valid. Rastegin extended this result to the case of mixed states and generalized quantum measurements [37, 66]. These works are mainly based on Riesz-Thorin theorem. The bound is not tight, except for $c=\frac{1}{\sqrt{N}}$ $[23,27]$.

$\diamond$ For $(\alpha, \beta) \in \underline{\mathcal{C}}$, the MU bound $\mathcal{B}^{\mathrm{MU}}(c)$ remains valid due to the decreasing property of Rényi entropy with the index. Here again, for $c=\frac{1}{\sqrt{N}}$ the bound is optimal $[23,27]$.

$\diamond$ For $(\alpha, \beta) \in \bar{C}$, the Deutsch bound $\mathcal{B}^{D}(c)$ remains valid. This result is due to MU who solved the minimization of the sum of min-entropies (infinite indices) subject to the Landau-Pollak inequality. Note that the Deutsch bound is valid in the whole positive quadrant (but it is not optimal) due to the decreasing property of the Rényi entropy versus the index.

$\diamond$ For $\beta=\alpha$, Puchała, Rudnicki and Życzkowski (PRZ) in [41] derived recently a series of $N-1$ bounds depending on the transformation matrix $T$ by using majorization technique. We denote by $\mathcal{B}_{\alpha ; \log }^{\mathrm{PRZ}}(T)$ the greatest of those bounds which is not $T$ optimal although it improves previous ones in several situations. A particular bound of 
the series (the worst one) depends only on the overlap $c$, and expresses as $\frac{1}{1-\alpha} \log \left[\left(\frac{1+c}{2}\right)^{2 \alpha}+\left(1-\left(\frac{1+c}{2}\right)^{2}\right)^{\alpha}\right]$ but it is not $c$-optimal. Further extensions of this work to mixed states and generalized quantum measurements are given by Friedland et al [42].

$\diamond$ For $(\alpha, \beta) \in[0,1]^{2}$, the $\mathrm{CP}$ bounds remain valid due to the decreasing property of Rényi entropy with the index.

$\diamond$ For $(\alpha, \beta) \in \mathbb{R}_{+}^{2}$ and $N=2$, we derived recently the $T$-optimal bound $\overline{\mathcal{B}}_{\alpha, \beta ; \log }(T)$. It depends only on the overlap, so that it is $c$-optimal as well, and $\overline{\mathcal{B}}_{\alpha, \beta ; \log }(T)=\widetilde{\mathcal{B}}_{\alpha, \beta ; \log ; 2}(c)$ [40]. Note that this equality is trivial since only $c$ parametrizes all the $\left|T_{i j}\right|$ and that in this case the phases play no role (due to the symmetry of the Bloch sphere or from the $Z-Y$ decomposition for a single qubit [40]). Numerical solutions have been found in the whole quadrant, and we have been able to derive analytical expressions in some regions. In addition, the states that correspond to the bound were obtained, in terms of the whole matrix $T$.

- Tsallis entropies:

$\diamond$ For $\beta=\alpha$ and pure states, the inequality

$$
S_{\alpha}(p(A, \rho))+S_{\alpha}(p(B, \rho))+(1-\alpha) S_{\alpha}(p(A, \rho)) S_{\alpha}(p(B, \rho)) \geqslant \frac{1-\left(\frac{1+c}{2}\right)^{2(\alpha-1)}}{\alpha-1}
$$

has been derived in [20]. This relation can be viewed as a consequence of the fact that the sum of Rényi entropies with equal indices is lower bounded by the Deutsch bound, together with relation (6) linking $S_{\alpha}$ and $R_{\alpha}$. This bound has been refined to $\frac{1-c^{2(\alpha-1)}}{\alpha-1}$ when $\alpha \in\left[\frac{1}{2} ; 1\right]$, starting from the MU inequality in the conjugacy curve, and using the decreasing property of $R_{\alpha}$ versus $\alpha$, and relation (6).

$\diamond$ For $(\alpha, \beta) \in \mathcal{C}$, following recent works of Rastegin [35, 36], one can obtain the inequality

$S_{\alpha}(p(A, \rho))+S_{\beta}(p(B, \rho)) \geqslant \frac{1-c^{2(\lambda-1)}}{\lambda-1} \equiv \mathcal{B}_{\alpha, \beta ; \text { id }-1}^{R}(c) \quad$ with $\quad \lambda=\max \{\alpha, \beta\}$

(id stands for the identity function, $\operatorname{id}(x)=x$ ).

$\diamond$ For $(\alpha, \beta) \in \underline{\mathcal{C}}$, bound $\mathcal{B}_{\alpha, \beta \text {; id }-1}^{R}(c)$ remains valid due to the decreasing property of Tsallis entropy versus the entropic index.

$\diamond$ For $(\alpha, \beta) \in[0,1]^{2}$, MU, Deutsch and CP bounds remain valid due to the decreasing property of Tsallis entropy with the index.

One can find in the literature many bounds improving the above mentioned, in special contexts (particular overlap and/or particular pair of indices). We refer the interested reader to $[21,25,30,31,35,37,39,43,45]$. For the sake of completeness of this short review, it is worth mentioning that there is a new insight of EURs that allows the observer to have access to a quantum memory [32, 33, 38, 67-69]. Also, there exist entropic formulations of the UP for more than two measurements (in particular, for mutually unbiased bases) [19, 28, 29, 70, 71] and for observables with continuous spectra [23, 72-74]. These topics have many applications in different issues of quantum information such that entanglement detection, proof of the security of quantum cryptographic protocols, and others [75-80]. Such studies go beyond the scope of the present paper.

Finally, it can be shown that some bounds and relations discussed above can be expressed in terms of the generalized entropies of the family $F_{\lambda}$ (with a common function $f$ for both entropies, but any pair of entropic indices): 
- $F_{\lambda}$ entropies:

$\diamond$ For $(\alpha, \beta) \in \mathcal{C} \cup \underline{\mathcal{C}}$, with the additional condition that $x f^{\prime}(x)$ is increasing, following the same approach as that of Rastegin in $[35,36]$ and using the decreasing property of $F_{\lambda}$ versus $\lambda$, one can prove the relation

$$
F_{\alpha}(p(A, \rho))+F_{\beta}(p(B, \rho)) \geqslant \frac{f\left(c^{2(\lambda-1)}\right)}{1-\lambda} \equiv \mathcal{B}_{\alpha, \beta ; f}^{R}(c) \quad \text { with } \lambda=\max \{\alpha, \beta\},
$$

which includes as particular cases the results of MU and of Rastegin.

$\diamond$ For $\beta=\alpha \geqslant 1$ : since $F_{\alpha}$ is Schur-concave, the corollary 2 of [41] allows us to derive a $T$-dependent bound for $F_{\alpha}(p(A, \rho) \otimes p(B, \rho))$, where $\otimes$ denotes the Kronecker product $^{4}$. If $f(x)+f(y) \leqslant f(x y)$ for $0 \leqslant x, y \leqslant 1$ then $F_{\alpha}(p(A, \rho))+F_{\alpha}(p(B, \rho)) \geqslant$ $F_{\alpha}(p(A, \rho) \otimes p(B, \rho))$. Applying the results of PRZ to the right-hand side we obtain a bound for the sum of $F_{\lambda}$ entropies. Rényi and Tsallis entropies with entropic index greater than or equal to one are particular cases.

$\diamond$ For $\beta=\alpha \leqslant 1$ : from the Schur concavity of $F_{\lambda}$ we have again a $T$-dependent bound for $F_{\alpha}(p(A, \rho) \otimes p(B, \rho))$. Now, if $f(x)+f(y) \geqslant f(x y)$ for $x, y \geqslant 1$, one has $F_{\alpha}(p(A, \rho))+F_{\alpha}(p(B, \rho)) \geqslant F_{\alpha}(p(A, \rho) \otimes p(B, \rho))$ (notice that Tsallis entropy does not fulfill this property in this case). Therefore, PRZ results applied to the right-hand side allows again to obtain a bound for the sum of this class of entropies. Rényi entropies with entropic index lower than or equal to one are particular cases.

$\diamond$ For $(\alpha, \beta) \in[0,1]^{2}, \mathrm{MU}$, Deutsch and CP bounds remain valid due to the decreasing property of the entropy $F_{\lambda}$ with the index.

\section{Generalized EURs}

We extend results summarized in the preceding section for POVM pairs, and generalized entropies (1) with arbitrary pairs of entropic functionals $\left(h_{A}, \phi_{A}\right)$ and $\left(h_{B}, \phi_{B}\right)$. Our approach follows that of de Vicente and Sánchez-Ruiz [26] except that here the concomitant optimization problem is mainly solved in a geometrical way. This allows us to generalize the results to arbitrary entropic functionals. Moreover, we use the fact that the Landau-Pollak inequality applies for POVM pairs and for both pure and mixed states $[81,82]$ to argue that our results include these situations.

Our major results are given by the following proposition, and corollaries 2-4:

Proposition 1. Let us consider a pair of POVM $A=\left\{A_{i}\right\}_{i=1}^{N_{A}}$ and $B=\left\{B_{j}\right\}_{j=1}^{N_{B}}$ acting on an $\mathrm{N}$-dimensional Hilbert space $\mathcal{H}$, and consider a quantum system described by a density operator $\rho$ acting on $\mathcal{H}$. Then for generalized entropies of the form (1), with any two pairs of entropic functionals $\left(h_{A}, \phi_{A}\right)$ and $\left(h_{B}, \phi_{B}\right)$, the following uncertainty relation holds:

$$
H_{\left(h_{A}, \phi_{A}\right)}(p(A, \rho))+H_{\left(h_{B}, \phi_{B}\right)}(p(B, \rho)) \geqslant \mathcal{B}_{\left(h_{A}, \phi_{A}\right),\left(h_{B}, \phi_{B}\right)}(c(A, B)),
$$

where the overlap triplet $c(A, B)=\left(c_{A}, c_{B}, c_{A, B}\right)$ is given by equation (13), and the lower bound expresses as

${ }^{4}\left[p_{1} \cdots p_{N}\right]^{t} \otimes\left[q_{1} \cdots q_{M}\right]^{t}=\left[p_{1} q_{1} \cdots p_{1} q_{M} \cdots p_{N} q_{1} \cdots p_{N} q_{M}\right]^{t}$ 
$\mathcal{B}_{\left(h_{A}, \phi_{A}\right),\left(h_{B}, \phi_{B}\right)}(\boldsymbol{c})=\left\{\begin{array}{l}\mathcal{D}_{\left(h_{A}, \phi_{A}\right)}\left(\gamma_{A}\right)+\mathcal{D}_{\left(h_{B}, \phi_{B}\right)}\left(\gamma_{B}\right) \quad \text { if } \gamma_{A, B} \leqslant \gamma_{A}+\gamma_{B} \\ \min _{\theta \in\left[\gamma_{A}, \gamma_{A, B}-\gamma_{B}\right]}\left(\mathcal{D}_{\left(h_{A}, \phi_{A}\right)}(\theta)+\mathcal{D}_{\left(h_{B}, \phi_{B}\right)}\left(\gamma_{A, B}-\theta\right)\right) \text { otherwise }\end{array}\right.$

with

$$
\gamma_{A} \equiv \arccos c_{A}, \quad \gamma_{B} \equiv \arccos c_{B}, \quad \gamma_{A, B} \equiv \arccos c_{A, B}
$$

and

$$
\mathcal{D}_{(h, \phi)}(\theta) \equiv h\left(\left\lfloor\frac{1}{\cos ^{2} \theta}\right\rfloor \phi\left(\cos ^{2} \theta\right)+\phi\left(1-\left\lfloor\frac{1}{\cos ^{2} \theta}\right\rfloor \cos ^{2} \theta\right)\right),
$$

where $\lfloor\cdot\rfloor$ indicates the floor part.

Proof. See appendix A.

For the sake of simplicity, when dealing with $F_{\lambda}$ entropies (with the same function $f$ for both observables), the bound is simply denoted

$$
\mathcal{B}_{\alpha, \beta ; f} \equiv \mathcal{B}\left(\frac{f}{1-\alpha}, \mathrm{id}^{\alpha}\right),\left(\frac{f}{1-\beta}, \mathrm{id}^{\beta}\right) \cdot
$$

Let us note the following facts:

- $\mathcal{B}_{\left(h_{A}, \phi_{A}\right),\left(h_{B}, \phi_{B}\right)}(\boldsymbol{c})$ is explicitly independent of $\boldsymbol{N}=\left(N_{A}, N_{B}, N\right)$.

- Previous results in the literature, in particular those of de Vicente and Sánchez-Ruiz [26], are extended here from Shannon to more general $(h, \phi)$-entropies, the former being recovered as a particular case. Moreover, our result applies in the POVM framework and for both pure and mixed states.

- For Tsallis entropies with $\beta=\alpha$, it is straightforward to obtain relations of the type

$S_{\alpha}(p(A, \rho))+S_{\alpha}(p(B, \rho))+(1-\alpha) S_{\alpha}(p(A, \rho)) S_{\alpha}(p(B, \rho)) \geqslant \frac{1-\mathrm{e}^{(1-\alpha) B_{\alpha, \alpha ; \log }(\boldsymbol{c})}}{\alpha-1}$

that improve and generalize the findings in [20] and is valid for all positive entropic index.

Note that, except when $\gamma_{A, B} \leqslant \gamma_{A}+\gamma_{B}$, bound (17) is implicit. This is also the case for several bounds in the literature [26, 41, 44]. But, as for [26, 44], the problem is shown to be reduced to an optimization on one parameter over a bounded interval, instead of on $N(N-2)$ parameters. Notice that from the increasing property of $\mathcal{D}_{(h, \phi)}(\theta)$ versus $\theta$ (see appendix A), an explicit lower bound can be obtained:

Corollary 1. Whatever the overlaps triplet be, bound (17) satisfies

$$
\mathcal{B}_{\left(h_{A}, \phi_{A}\right),\left(h_{B}, \phi_{B}\right)}(\boldsymbol{c}) \geqslant \mathcal{D}_{\left(h_{A}, \phi_{A}\right)}\left(\gamma_{A}\right)+\mathcal{D}_{\left(h_{B}, \phi_{B}\right)}\left(\gamma_{B}\right) \text {. }
$$

Thus the expression on the right hand side lower bounds the entropy sum even when $\gamma_{A, B}>\gamma_{A}+\gamma_{B}$

Note however that this analytic bound is weaker, and that when $\gamma_{A}=\gamma_{B}=0$ it turns out to be trivial.

Finally, it is to be noticed that bound (17) is in general not $c$-optimal. Indeed, our method for solving the minimization problem first treats separately the contribution of each observable in the entropy sum and, only in a second step the link between the observables is taken into account through the Landau-Pollak inequality. In some specific cases, this relative weakness disappears, as we see now. 
Hereafter, we consider the case of nondegenerate quantum observables. In this case, we have $N_{A}=N_{B}=N, c_{A}=c_{B}=1\left(\gamma_{A}=\gamma_{B}=0\right)$ and $c_{A, B}=c\left(\gamma_{A, B}>0\right.$ except when $\left.c=1\right)$, then the bound (17) reduces to

$$
\mathcal{B}_{\left(h_{A}, \phi_{A}\right),\left(h_{B}, \phi_{B}\right)}(c)=\min _{\theta \in[0, \gamma]}\left(\mathcal{D}_{\left(h_{A}, \phi_{A}\right)}(\theta)+\mathcal{D}_{\left(h_{B}, \phi_{B}\right)}(\gamma-\theta)\right)
$$

with $\gamma=\arccos c$.

As already mentioned, bound (22) is in general not $c$-optimal. However, it can be shown that this bound does turn out to be optimal for some particular values of the overlap. This is summarized in the following corollary:

Corollary 2. When $c>\frac{1}{\sqrt{2}}$ and $N=2$ or $N \geqslant 4$, the bound (22) is c-optimal,

$\widetilde{\mathcal{B}}_{\left(h_{A}, \phi_{A}\right),\left(h_{B}, \phi_{B}\right) ; N}(c)=\widetilde{\mathcal{B}}_{\left(h_{A}, \phi_{A}\right),\left(h_{B}, \phi_{B}\right) ; 2}(c)=\min _{\theta \in[0 ; \gamma]}\left(\mathcal{D}_{\left(h_{A}, \phi_{A}\right)}(\theta)+\mathcal{D}_{\left(h_{B}, \phi_{B}\right)}(\gamma-\theta)\right)$.

Proof. See appendix B.

We suspect that this corollary is also valid when $N=3$, but we have not been able to prove it yet.

A consequence of the corollary is that, in the range of the overlap $c>\frac{1}{\sqrt{2}}$, the bound (22) reduces to that of the qubit case and improves all $c$-dependent bounds such as those of MU [6] or Rastegin $[35,36]$ in the context of entropies of the $F_{\lambda}$ family. In particular, since $\mathcal{B}^{\mathrm{MU}}$ and $\mathcal{B}_{\alpha, \beta ; f}^{R}(c)$ do not depend on $N$, then $\mathcal{B}_{\alpha, \beta ; \log }(c) \geqslant \mathcal{B}^{\mathrm{MU}}$ and $\mathcal{B}_{\alpha, \beta ; f}(c) \geqslant \mathcal{B}_{\alpha, \beta ; f}^{R}(c)$ for any $c \geqslant \frac{1}{\sqrt{2}}$ and any $N \geqslant 2$. Moreover, it is shown in [40] that, for a certain range of entropic indices and in the context of Rényi entropies, this $c$-optimal bound takes an analytical expression.

Now, we particularize the proposition to the case of Rényi entropy [setting $\phi(x)=x^{\lambda}$ and $f(x)=\frac{\log x}{1-\lambda}$, i.e., $f=\log$ in the $F_{\lambda}$ family], which is mostly used in the literature of EURs, and compare our bound with previous ones, as we detail in the following two corollaries:

Corollary 3. In the context of Rényi entropy, the bound (22) is higher than that of Deutsch:

$$
\mathcal{B}_{\alpha, \beta ; \log }(c) \geqslant \mathcal{B}^{D}(c)=-2 \log \left(\frac{1+c}{2}\right) \text {. }
$$

Proof. See appendix C.

This result is particularly interesting above the conjugacy curve, $(\alpha, \beta) \in \bar{C}$, where the only $c$-dependent explicitly known bound for Rényi entropies is precisely $\mathcal{B}^{D}(c)$.

It is known that the sum of Rényi entropies below the conjugacy curve, $(\alpha, \beta) \in \underline{\mathcal{C}}$, is lower bounded by MU result. For $c>\frac{1}{\sqrt{2}}$ we were able to improve this bound, but for $c \leqslant \frac{1}{\sqrt{2}}$ it is not always the case. Indeed, we have:

Corollary 4. In the context of Rényi entropy, when $c \leqslant \frac{1}{2}$ and $(\alpha, \beta) \in \underline{\mathcal{C}}$, the bound (22) is lower than that of $M U$ : 


$$
\mathcal{B}_{\alpha, \beta ; \log }(c) \leqslant \mathcal{B}^{\mathrm{MU}}(c)=-2 \log c .
$$

Proof. See appendix D.

To the best of our knowledge, in the range of the overlap $c \leqslant \frac{1}{2}$, the MU result is the tightest $c$-dependent bound when $(\alpha, \beta) \in \underline{\mathcal{C}}$.

\section{Comparison with previously known bounds}

\subsection{MU, Rastegin and CP bounds}

We now compare our bound with previously known ones in the nondegenerate context, for Rényi and Tsallis entropies with indices $(\alpha, \beta)$ in the region $\mathcal{C} \cup \underline{\mathcal{C}}$ or just within $[0 ; 1]^{2}$. Relative differences are shown through density plots in figures $2-5$, for chosen typical values of the overlap $c$. Positivity of these differences indicates that our bound improves the previous.

In figure 2 we plot $\frac{\mathcal{B}_{\alpha, \beta ;} \log (c)-\mathcal{B}^{\mathrm{MU}}(c)}{\mathcal{B}_{\alpha, \beta ;} \log (c)}$ for entropic indices in and below the conjugacy curve, $(\alpha, \beta) \in \mathcal{C} \cup \underline{\mathcal{C}}$. We observe the following behavior of our bound with respect to MU result:

- Up to $c=\frac{1}{2}$ ( $c=0.5$ is shown), the relative difference is negative or zero, so our bound does not improve the MU one (corollary 4).

- When $c$ is between $\frac{1}{2}$ and $\frac{1}{\sqrt{2}}(c=0.706$ is shown), the relative difference is positive or negative (although very small), so our bound improves the MU one in some regions of the $\alpha-\beta$-plane. This region is delimited by the white line: the improvement takes place below this curve; we observe that the region of improvement increases with the overlap. - When $c$ exceeds $\frac{1}{\sqrt{2}}(c=0.708$ and 0.9 are shown), the relative difference is positive, so our bound improves MU one (corollary 2); the improvement significantly increases with the overlap.

In figure 3 we plot the relative difference: $\frac{\mathcal{B}_{\alpha, \beta ; \text { id }-1}(c) \mathcal{B}_{\alpha, \beta}^{R} \text {; id - } 1(c)}{\mathcal{B}_{\alpha, \beta ; \text {; } 1-1}(c)}$ for entropic indices in and below the conjugacy curve, $(\alpha, \beta) \in \mathcal{C} \cup \underline{\mathcal{C}}$. We observe the following behavior with respect to Rastegin results:

- Up to $c=\frac{1}{\sqrt{2}}$ ( $c=0.5$ and 0.6 are shown), the relative difference is positive or negative, so our bound improves the Rastegin one in some regions of the $\alpha-\beta$-plane. The regions where an improvement occurs are outside the domain marked by the black line. These regions always exist (even when $c<\frac{1}{2}$ ) and increase with the overlap.

- When $c$ exceeds $\frac{1}{\sqrt{2}}(c=0.708$ and 0.9 are shown), the relative difference is positive, so our bound improves Rastegin one (corollary 2) and the improvement increases significantly with the overlap.

In figures 4 and 5 we plot the relative differences: $\frac{\mathcal{B}_{\alpha, \beta f}(c)-\beta^{\mathrm{CP}}{ }^{\star}(c)}{\beta_{\alpha, \beta f}(c)}$, for $f=\mathrm{id}$ and $\log$, respectively, where $\mathcal{B}^{\mathrm{CP}^{\star}}(c)=\mathcal{B}^{\mathrm{CP}}\left(c, c_{2}\right)$ with $c_{2}=\frac{\sqrt{N-2+c^{2}}}{N-1}$ being the lowest possible second larger value of the $\left|T_{i j}\right|$ (we choose here $N=3$ and $N=10$ respectively); the entropic indices are $(\alpha, \beta) \in[0 ; 1]^{2}$. We observe the following behavior with respect to $\mathrm{CP}$ results: 


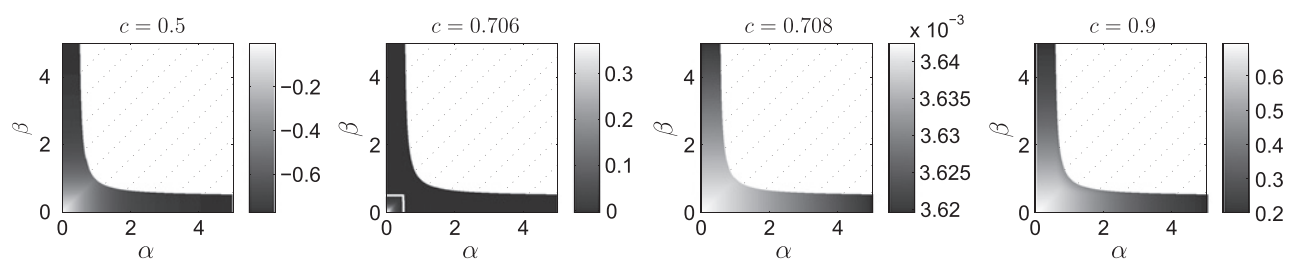

Figure 2. Rényi entropy case: density plots of $\frac{\mathcal{B}_{\alpha, \beta ;} \log (c)-\mathcal{B}^{\mathrm{MU}}(c)}{\mathcal{B}_{\alpha, \beta ;} \log (c)}$, for $(\alpha, \beta) \in \mathcal{C} \cup \underline{\mathcal{C}}$ when $c=0.5,0.706,0.708$ and 0.9 .
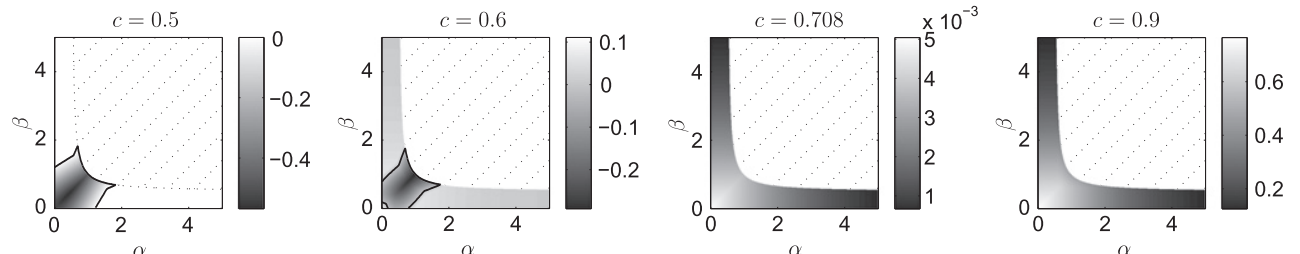

Figure 3. Tsallis entropy case: density plots of $\frac{\mathcal{B}_{\alpha, \beta ; \mathrm{id}-1}(c)-\mathcal{B}_{\alpha, \beta ; \mathrm{id}-1}(c)}{\mathcal{B}_{\alpha, \beta ; \mathrm{id}-1}(c)}$, for $(\alpha, \beta) \in \mathcal{C} \cup \underline{\mathcal{C}}$ when $c=0.5,0.6,0.708$ and 0.9 .
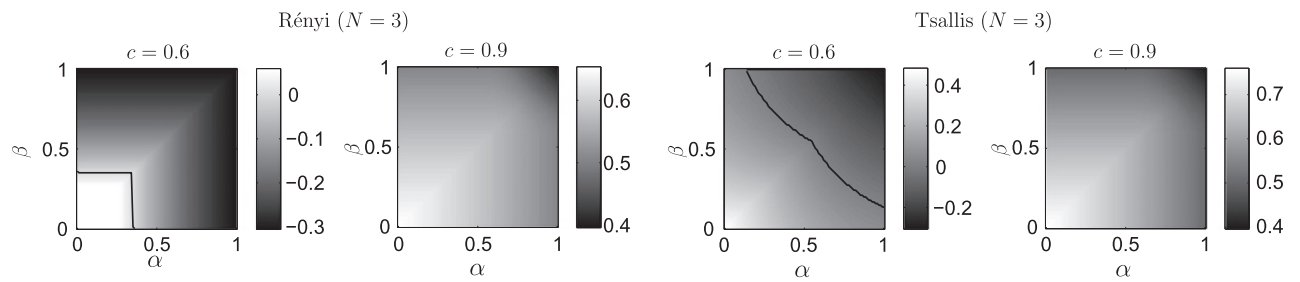

Figure 4. Rényi and Tsallis entropy cases for $N=3$ : density plots of $\frac{\mathcal{B}_{\alpha, \beta ; f}(c)-\mathcal{B}^{\mathrm{CP}^{*}}(c)}{\mathcal{B}_{\alpha, \beta ;}(c)}$, for $(\alpha, \beta) \in[0 ; 1]^{2}$ when $c=0.6$ and 0.9 .
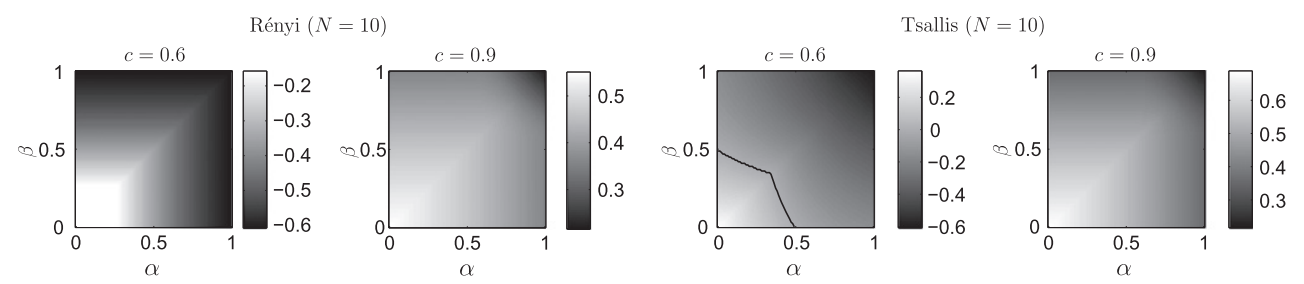

Figure 5. Same as figure 4 for $N=10$.

- For any value of $c$, the relative difference can be positive or negative, so our bound improves the $\mathrm{CP}$ one in some regions of the $\alpha-\beta$-plane. The regions where an improvement occurs are below the domain marked by the solid line in figures 4 and 5 . These regions generally exist (even when $c<\frac{1}{2}$ ) and their extension is greater with the overlap (the improvement always exists for $c \geqslant \frac{1}{\sqrt{2}}$ ).

- When $N$ increases (and $c<\frac{1}{\sqrt{2}}$ ), the domain of improvement is smaller. Remind however that the best possible $\mathrm{CP}$ bound $\mathcal{B}^{\mathrm{CP}}$ is plotted here. 

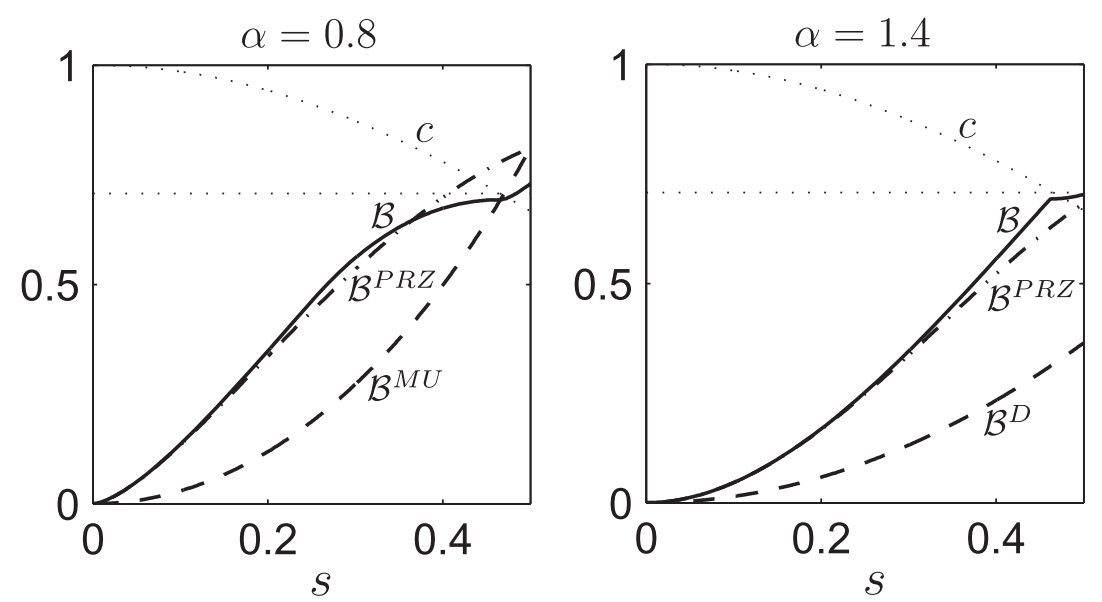

Figure 6. Rényi entropy case: bounds $\mathcal{B} \equiv \mathcal{B}_{\alpha, \alpha ; \log }(c)$ (solid line), $\mathcal{B}^{\mathrm{PRZ}} \equiv \mathcal{B}_{\alpha ; \log }^{\mathrm{PRZ}}(T)$ (dashed-dotted line), $\mathcal{B}^{\mathrm{MU}} \equiv \mathcal{B}^{\mathrm{MU}}(c)$ (left plot, dashed line) and $\mathcal{B}^{D} \equiv \mathcal{B}^{D}(c)$ (right plot, dashed line), in terms of the power $s$ in the transformation matrix for $\alpha=0.8$ and 1.4. In addition, we plot the overlap $c$ in terms of $s$ (dotted line).
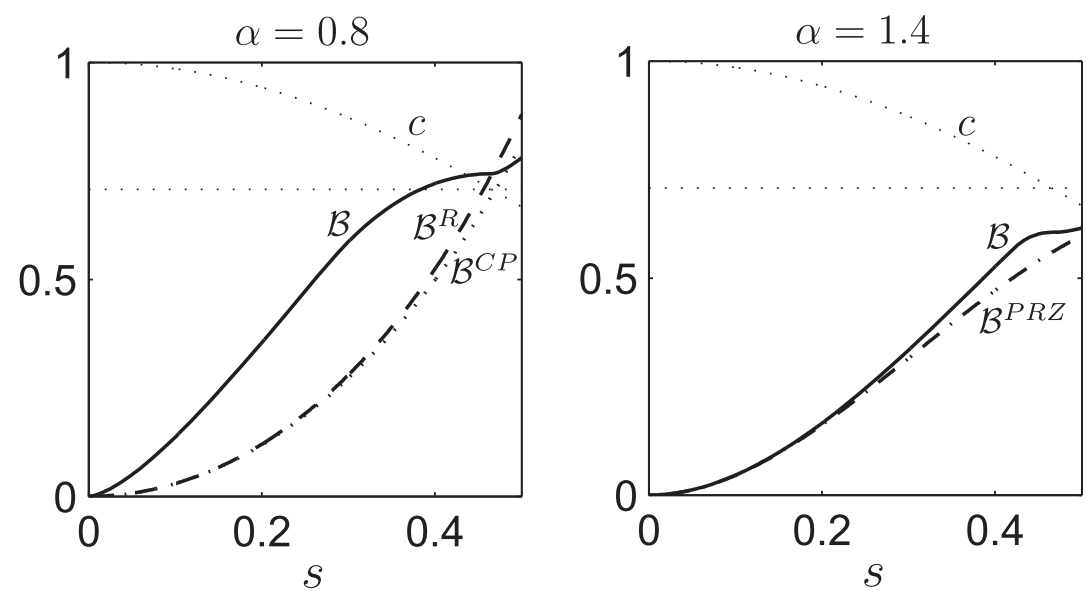

Figure 7. Tsallis entropy case: bounds $\mathcal{B}_{\alpha, \alpha ; \text { id }-1}(c)$ (solid line), $\mathcal{B}_{\alpha, \alpha \text {; id - } 1}^{R}(c)$ (left plot, dashed line), $\mathcal{B}^{\mathrm{CP}}(T)$ (left plot, dotted line below that of $\left.\mathcal{B}_{\alpha, \alpha \text {; id - } 1}^{R}(c)\right), \mathcal{B}_{\alpha ; \text { id }-1}^{\mathrm{PRZ}}(T)$ (right plot, dashed-dotted line), in terms of the power $s$ in the transformation matrix for $\alpha=0.8$ and 1.4. In addition, we plot the overlap $c$ in terms of $s$ (dotted line).

\subsection{Bounds for powers of a circular permutation matrix in the line $\beta=\alpha$}

An illustrative example to consider for the evaluation of generalized EURs is given in [41], where a special class of transformation matrices is used. Indeed, the quantum observables here are such that the transformation between their eigenbases is a power of a circular $N$-dimensional permutation matrix, namely $T_{N}(s)=\left[\begin{array}{cc}0 & I_{N-1} \\ 1 & 0 \cdots 0\end{array}\right]^{s}$ with $s \in\left[0 ; \frac{1}{2}\right]$ and where $I_{N-1}$ denotes the $(N-1) \times(N-1)$ identity matrix. We compute our bound in these cases for $N=3$ and for some chosen, equal entropic indices, and we compare our results with 

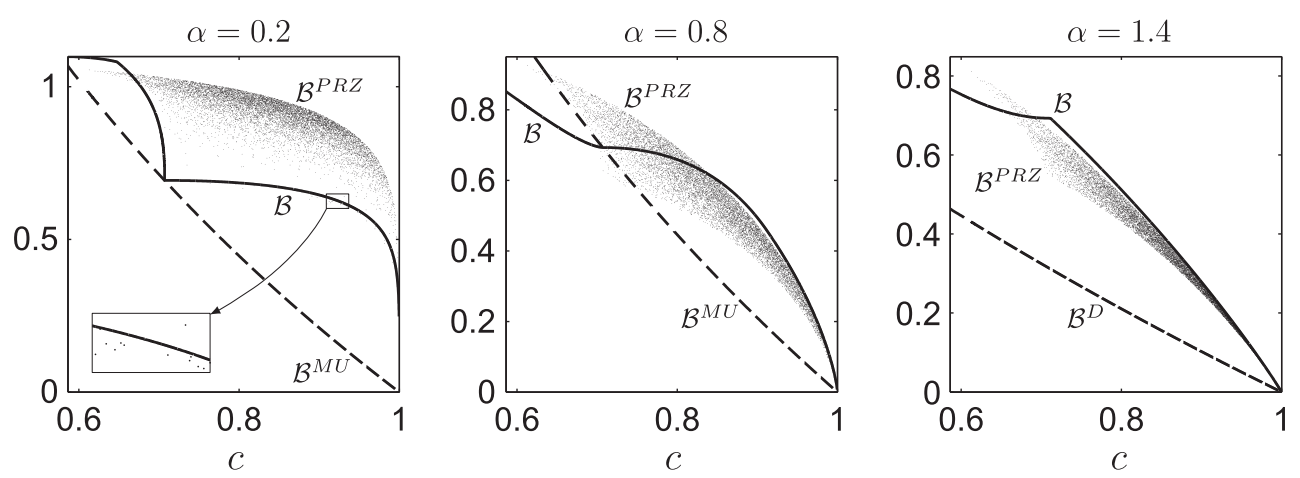

Figure 8. Rényi entropy case: bounds $\mathcal{B}_{\alpha, \alpha}$; log $(c)$ (solid line), $\mathcal{B}^{\mathrm{MU}}(c)$ (dashed line, left and middle plots), $\mathcal{B}_{\alpha ; \log }^{\mathrm{PRZ}}(T)$ (dots), and $\mathcal{B}^{D}(c)$ (dashed line, right plot), in terms of the overlap $c$ for $\alpha=0.2,0.8$ and 1.4 .

the bounds of PRZ, MU and Deutsch in the case of Rényi entropy (figure 6), and with the bounds of Rastegin, CP and PRZ in the case of Tsallis entropy (figure 7). In this particular example, $T_{N}(s)$ can be analytically determined, allowing for an analytic expression for both $\mathrm{CP}$ bounds $\mathcal{B}^{\mathrm{CP}}$ and $\mathcal{B}^{\overline{\mathrm{CP}}}$. It appears that, whatever $N$, both bounds coincide and that they coincide with the MU bound.

In figure 6 we plot the bounds $\mathcal{B}_{\alpha, \alpha ; \log }(c), \mathcal{B}_{\alpha ; \log }^{\mathrm{PRZ}}(T), \mathcal{B}^{\mathrm{MU}}(c)$ and $\mathcal{B}^{D}(c)$ for the Rényi entropic formulation of the UP, in terms of the power $s$ in the transformation matrix, when $\alpha=0.8$ and 1.4. The overlap $c=c(s)$ corresponding to the transformation $T=T(s)$ is also shown in the figure. We observe that:

- For $\alpha=0.8$ our bound improves both PRZ and MU ones for a wide range of values of $s$. The fact that our bound can be lower than that of PRZ for $c>\frac{1}{\sqrt{2}}$ does not contradict corollary 2. Indeed, the PRZ bound is $T$-dependent and is evaluated here for a particular $T$; it is not the minimum over all $T$ for a given $c$.

- For $\alpha=1.4$ our bound improves Deutsch result (corollary 3) as well as PRZ for all $s$.

In figure 7 we plot the bounds $\mathcal{B}_{\alpha, \alpha ; \text { id }-1}(c), \mathcal{B}_{\alpha ; \alpha ; \text { id }-1}^{R}(c), \mathcal{B}^{\overline{\mathrm{CP}}}=\mathcal{B}^{\mathrm{CP}}=\mathcal{B}^{\mathrm{MU}}$, and $\mathcal{B}_{\alpha}^{\mathrm{PRZ} \text {; }-1}(T)$, for the Tsallis entropic formulation of the UP, in terms of the power $s$ in the transformation matrix, when $\alpha=0.8$ and 1.4. We observe that:

- For $\alpha=0.8$ our bound improves both CP and Rastegin ones in a wide range of values of $s$.

- For $\alpha=1.4$ our bound improves PRZ one for all $s$.

\subsection{Bounds for randomly drawn unitary matrices in the line $\beta=\alpha$}

As a further example, we randomly generate $10^{4}$ unitary matrices $T$ sampled according to a Haar (uniform) distribution on $\mathcal{V}(3)[83,84]$. We compute our bound in these cases for some chosen, equal entropic indices, and we compare our results with the bounds of PRZ, MU and Deutsch in the case of Rényi entropy (figure 8), with the bounds of Rastegin and PRZ in the case of Tsallis entropy (figure 9), and with $\mathcal{B}^{\overline{\mathrm{CP}}}$ in both cases (figure 10). 

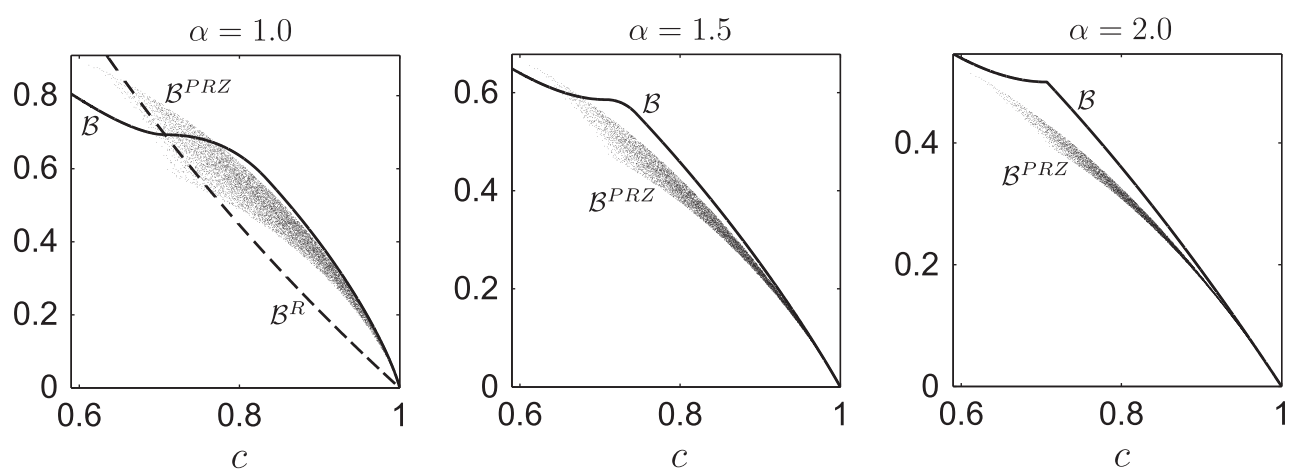

Figure 9. Tsallis entropy case: bounds $\mathcal{B}_{\alpha, \alpha ; \text { id -1 }}(c)$ (solid line), $\mathcal{B}_{\alpha, \alpha \text {; id - } 1}^{R}(c)$ (dashed line, left plot), and $\mathcal{B}_{\alpha ; \log }^{\mathrm{PRZ}}(T)$ (dots), in terms of the overlap $c$ for $\alpha=1,1.5$ and 2.
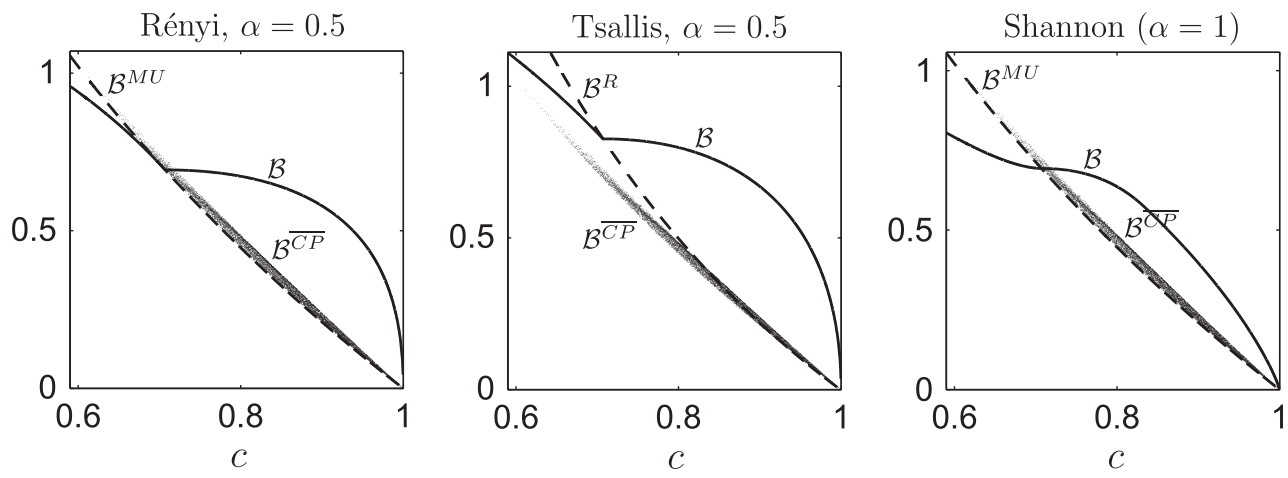

Figure 10. Rényi, Tsallis and Shannon entropy cases: bounds $\mathcal{B}_{\alpha, \alpha ; f}(c)$ (solid line), $\mathcal{B}^{\mathrm{MU}}(c)$ or $\mathcal{B}_{\alpha, \alpha \text {; id }-1}^{R}$ (dashed line), $\mathcal{B}^{\overline{\mathrm{CP}}}(T)$ (dots), in terms of the overlap $c$ for $\alpha=0.5$ (Rényi and Tsallis) and 1 (Shannon).

In figure 8 we plot the bounds $\mathcal{B}_{\alpha, \alpha ; \log }(c), \mathcal{B}^{\mathrm{MU}}(c), \mathcal{B}_{\alpha ; \log }^{\mathrm{PRZ}}(T)$, and $\mathcal{B}^{D}(c)$ for the Rényi entropic formulation of the UP, in terms of the overlap $c \geqslant \frac{1}{\sqrt{3}}$, when $\alpha=0.2,0.8$ and 1.4. We observe that:

- For $\alpha=0.2$, our bound improves MU one in the whole range of the overlap. We find transformation matrices such that our bound improves PRZ one, although with a low frequency of occurrence.

- For $\alpha=0.8$, our bound improves MU one when $c \geqslant \frac{1}{\sqrt{2}}$ (corollary2). We find transformation matrices such that our bound improves PRZ one, with a frequency higher than for $\alpha=0.2$ and increasing with $c$ as well.

- For $\alpha=1.4$, our bound improves Deutsch one in the whole range of the overlap (corollary 3). Again, we find transformation matrices such that our bound improves PRZ one, with a frequency higher than for $\alpha=0.8$ and increasing with $c$ as well.

In figure 9 we plot the bounds $\mathcal{B}_{\alpha, \alpha ; \log }(c), \mathcal{B}_{\alpha, \alpha ; \text { id }-1}^{R}(c)$, and $\mathcal{B}_{\alpha ; \log }^{\mathrm{PRZ}}(T)$ for the Tsallis entropic formulation of the UP, in terms of the overlap $c \geqslant \frac{1}{\sqrt{3}}$, when $\alpha=1,1.5$ and 2 . We observe that: 
- For $\alpha=1$, our bound improves Rastegin one when $c \geqslant \frac{1}{\sqrt{2}}$ (corollary 2). We find transformation matrices such that our bound improves $P R Z$ one, with relatively high frequency of occurrence.

- For $\alpha=1.5$, we find transformation matrices such that our bound improves PRZ one in a wider range for the overlap and with higher frequency than for $\alpha=1$.

- For $\alpha=2$, for all the sampled matrices we find that our bound improves PRZ one in the whole range of the overlap.

In figure 10 we plot the bounds $\mathcal{B}_{\alpha, \alpha ; f}(c), \mathcal{B}^{\mathrm{MU}}(c)$ or $\mathcal{B}_{\alpha, \alpha \text {; id - } 1}^{R}(c)$, and $\mathcal{B}^{\overline{\mathrm{CP}}}(T)$ for both Rényi and Tsallis entropic formulation of the UP, in terms of the overlap $c \geqslant \frac{1}{\sqrt{3}}$, when $\alpha=0.5$ and 1 . We observe that:

- For any $\alpha$, our bound improves $\mathcal{B}^{\overline{\mathrm{CP}}}$ in a wide range of the overlap $c$.

- In the Tsallis context, for $\alpha \leqslant \frac{1}{2}$, for all the sampled matrices, we find an improvement of $\mathcal{B}^{\overline{\mathrm{CP}}}$ in the whole range of the overlap. We observe that the range of values of $c$ for which an improvement of the CP bound occurs, decreases with $\alpha$.

We notice that, as MU, Deutsch, Rastegin and our bounds depend only on the overlap $c$, then the same relative behaviors remain valid for dimensions higher than 3 (at least for $\left.c \geqslant \frac{1}{\sqrt{3}}\right)$. In contrast, that may not be the case for the relation between CP, PRZ and our bound, since the formers depend on the whole transformation matrix $T$; indeed, we expect an increase of the predominance of PRZ and CP over other $c$-dependent bounds. However, our bound is easier to calculate than PRZ one for instance whose computation complexity increases combinatorially with the dimension of the matrix $T$.

\section{Concluding remarks}

In this contribution we provide a general entropy-like formulation of the UP, for any pair of POVM in the case of pure or mixed states in finite dimensions. The sum of generalized $(h, \phi)$-entropies (1) associated to two POVM sets is proposed as measure of joint uncertainty, and lower bounds for that sum are searched for, in terms of the overlaps $c$ between the POVM, which in a sense quantifies the degree of incompatibility of the observables. Our main result is summarized in the proposition of section 3 , where we give a $c$-dependent lower bound for the entropy-sum, leading to the family of EURs (16). To obtain this, we follow the same approach as de Vicente and Sánchez-Ruiz appealing to the Landau-Pollak inequality, and we solve the concomitant constrained minimization problem, mainly in a geometrical manner. In this way, the calculation of a $c$-dependent bound reduces to the resolution of the straightforward one-dimensional minimization problem in (17).

Our uncertainty relation (16) generalizes previous similar results in several ways, namely, it is valid for:

- Salicrú generalized entropic forms (including Rényi (3) and Tsallis (4) entropies, which are obtained for $\phi(x)=x^{\lambda}$ with $h(x)=\frac{\log x}{1-\lambda}$ and $h(x)=\frac{x-1}{1-\lambda}$, respectively),

- any choice for the pair of entropic functionals $\left(h_{A}, \phi_{A}\right)$ and $\left(h_{B}, \phi_{B}\right)$ (overcoming the limitation due to the Riesz-Thorin theorem that involves conjugated pairs of indices when dealing with the family $F_{\lambda}(5)$ with the same $f$, which is mainly used in related literature),

- any pair of positive operator valued measures, and 
- both pure and mixed states (which is proved without recourse to the concavity property, that, for instance, Rényi entropy does not fulfill in general).

Besides we show that, in the case of nondegenerate quantum observables with overlap $c$, the bound reduces to the unidimensional minimization problem (22). Moreover, for values of the overlap greater than $\frac{1}{\sqrt{2}}$, our bound is $c$-optimal and it reduces to that of the qubit $(N=2)$ case (corollary 2). In other words, we improve all $c$-dependent bounds in that range of the overlap.

In addition, we go further in the case of Rényi entropies and we find that our bound improves Deutsch one in the whole range of values of the overlap (corollary 3), and also that our bound does not improve MU one for values of the overlap lower than or equal to $\frac{1}{2}$ (corollary 4). The former result is particularly interesting for entropic indices above the conjugacy curve where, up to our knowledge, Deutsch bound is the only known one with an analytic expression; whereas the latter result establishes that restricting the domain by the Landau-Pollak inequality, leads to a result weaker than using Riesz-Thorin theorem.

Finally, in section 4, we provide several examples that exhibit an improvement with respect to known results in the literature, in the cases of Rényi and Tsallis entropies.

The extension of our approach to take into account quantum memory and for more than two POVM sets is currently under investigation.

\section{Acknowledgments}

SZ and MP are very grateful to the Région Rhône-Alpes (France) for the grants that enabled this work. MP and GMB also acknowledge financial support from CONICET (Argentina), and warm hospitality during their stay at GIPSA-Lab. The authors thank Pr J-F Bercher for useful discussions about the class of Salicrú entropies. The authors acknowledge anonymous referees for helpful comments.

\section{Appendix A. Proof of the proposition}

Our aim is, given the probability vectors $p(A, \rho)$ and $p(B, \rho)$ associated with the POVM $A$ and $B$ respectively, to minimize the sum of $(h, \phi)$-entropies subject to the Landau-Pollak inequality. In this way, our method follows and advances on that of de Vicente and SánchezRuiz [26], and consists of two steps:

(i) Minimization of $H_{(h, \phi)}$ subject to $\max _{k} p_{k}=P$. At this step, the two sets of probabilities are treated separately. Thus, denoting by $H_{(h, \phi)}^{\min }(P)$ this minimal entropy, we arrive at the inequality $\quad H_{\left(h_{A}, \phi_{A}\right)}(p(A, \rho))+H_{\left(h_{B}, \phi_{B}\right)}(p(B, \rho)) \geqslant H_{\left(h_{A}, \phi_{A}\right)}^{\min }\left(P_{A, \rho}\right)+H_{\left(h_{B}, \phi_{B}\right)}^{\min }\left(P_{B, \rho}\right)$, where the right-hand side depends only on the two maximal probabilities.

(ii) Minimization of $H_{\left(h_{A}, \phi_{A}\right)}^{\min }\left(P_{A, \rho}\right)+H_{\left(h_{B}, \phi_{B}\right)}^{\min }\left(P_{B, \rho}\right)$ subject to the Landau-Pollak inequality. 


\section{A.1. First step: minimization of the $(h, \phi)$-entropy subject to a given maximum probability}

This problem involves looking for the vectors $p=\left[\begin{array}{lll}p_{1} & \ldots & p_{N}\end{array}\right]^{t} \in \mathcal{P}_{N}$ (the set of probability vectors in $\mathbb{R}_{+}^{N}$ ) that minimize a given $(h, \phi)$-entropy under the constraint that the maximum probability is provided ${ }^{5}$, i.e., we search for

$$
\min _{p \in \mathcal{P}_{N}} H_{(h, \phi)}(p)=\min _{p \in \mathcal{P}_{N}} h\left(\sum_{k=1}^{N} \phi\left(p_{k}\right)\right) \quad \text { s.t. } \quad \max _{k} p_{k}=P .
$$

Notice that, due to the normalization constraint, one necessarily has ${ }^{6}$

$$
P \in\left[\frac{1}{N} ; 1\right] \text {. }
$$

Note also that in the case $P=\frac{1}{N}$, then all the $p_{k}$ are equal to $\frac{1}{N}$ (uniform distribution) and thus the problem becomes trivial.

Using the fact that the function to be optimized is invariant under a permutation of the probability components, we can reduce the dimensionality of the problem in the following way: let us fix $p_{1} \equiv P$ and define $q=\left[\begin{array}{lll}q_{1} & \ldots & q_{N-1}\end{array}\right]^{t} \equiv\left[\begin{array}{lll}p_{2} & \ldots & p_{N}\end{array}\right]^{t}$; then, to solve the optimization problem (A.1) is equivalent to search for

$$
\left\{\begin{array}{l}
\min _{q \in \mathcal{P} \mathcal{T}_{P}} \varphi(q) \text { if } \phi \text { is concave, } \\
\max _{q \in \mathcal{P T}_{P}} \varphi(q) \text { if } \phi \text { is convex }
\end{array}\right.
$$

where we define

$$
\varphi(q)=\sum_{k=1}^{N-1} \phi\left(q_{k}\right)
$$

and we denote by $\mathcal{P} \mathcal{T}_{P}$ the allowed domain for $q$, i.e.

$\mathcal{P \mathcal { T }}_{P}=\left\{q \in \mathbb{R}^{N-1}: 0 \leqslant q_{k} \leqslant P \wedge \sum_{k=1}^{N-1} q_{k}=1-P\right\}=\mathcal{H C}_{P} \cap \mathcal{H P}_{P}$

with $\mathcal{H C}_{P}=[0 ; P]^{N-1}$ denoting an $(N-1)$-dimensional closed hypercube, and $\mathcal{H P}_{P}=\left\{q \in \mathbb{R}^{N-1}: \sum_{k=1}^{N-1} q_{k}=1-P\right\}$ corresponding to an $(N-2)$-dimensional hyperplane perpendicular to the vector $\mathbf{1}=\left[\begin{array}{lll}1 & \cdots & 1\end{array}\right]^{t}$. Notice that the point $\frac{1-P}{N-1}\left(\begin{array}{lll}1 & \cdots & 1\end{array}\right)$ is both inside the hypercube $\mathcal{H} \mathcal{C}_{P}$ and on the hyperplane $\mathcal{H} \mathcal{P}_{P}$, which guarantees that the intersection of those sets is not empty.

It can be seen that $\mathcal{P} \mathcal{T}_{P}$ is a convex polytope embedded in $\mathcal{H C}_{P}$ [87]; in other words, it is a convex body, convex hull of its vertices that are the pure points of this convex (i.e., the points that cannot be written as convex combination of several points of the set) [88, 89].

Next, since $\varphi$ is a strictly concave (respectively convex) function on $\mathbb{R}_{+}^{N-1}$, it is also concave (respectively convex) on the polytope $\mathcal{P T}_{P}$. It turns out that $\varphi$ achieves its minimum (respectively maximum) only on one or several of the extreme points (or pure points) of $\mathcal{P} \mathcal{T}_{P}$ $[62,90]$. The problem consists then in determining the set of pure points of (A.5). Before studying the case of arbitrary $N$, let us illustrate what happens in the cases $N=3$ and $N=4$

\footnotetext{
5 In the context of Shannon entropy, the problem was already solved in [85], using the Karush-Khun-Tucker sufficient conditions for convex optimization problems [62, 86].

${ }^{6}$ If $P<\frac{1}{N} \Rightarrow \sum_{k} p_{k} \leqslant N P<1$, which would contradict normalization.
} 

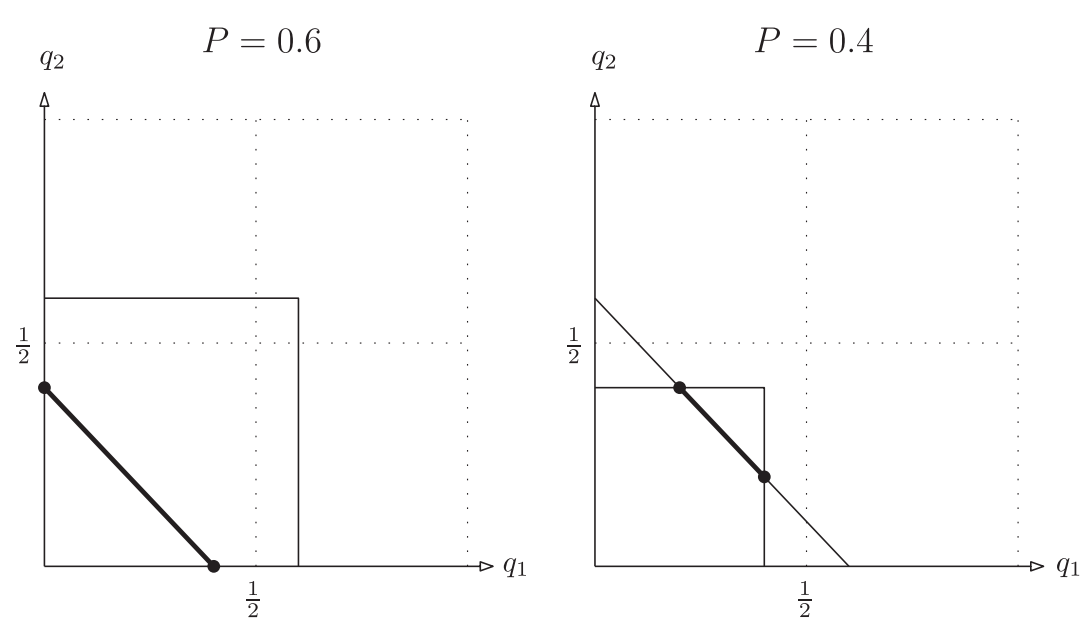

Figure A1. Domain $\mathcal{P} \mathcal{T}_{P}$ (line in bold) in the case $N=3$, for $P=0.6$ and 0.4 (from left to right). It is the intersection between the line $q_{1}+q_{2}=1-P$ and the square $[0 ; P]^{2}$. The pure points of $\mathcal{P} \mathcal{T}_{P}$ are given by the dots.
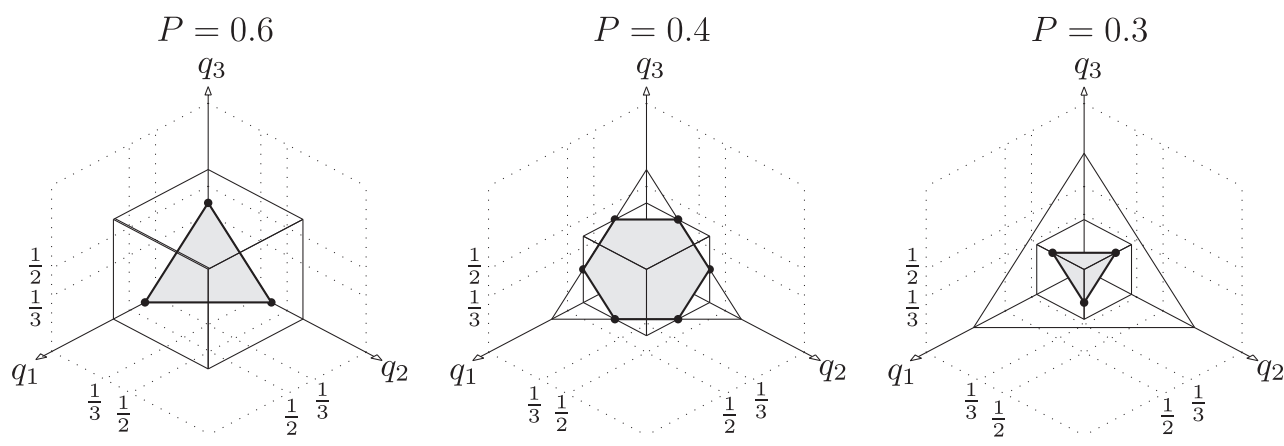

Figure A2. Domain $\mathcal{P \mathcal { T } _ { P }}$ (surface in gray) in the case $N=4$, for $P=0.6,0.4$ and 0.3 (from left to right). It is the intersection between the plane $q_{1}+q_{2}+q_{3}=1-P$ and the cube $[0 ; P]^{3}$. The border of the polytope $\mathcal{P} \mathcal{T}_{P}$ is represented by bold lines, and its pure points are given by the dots.

(the case $N=2$ is trivial since $\mathcal{P} \mathcal{T}_{P}$ reduces to the point $1-P$, and the maximizing probability vector is $(P, 1-P)$, where $P$ must be between $\frac{1}{2}$ and 1$)$.

A.1.1. Case $N=3$. Two different situations arise for the intersection of the line $q_{1}+q_{2}=1-P$ with the square $0 \leqslant q_{1} \leqslant P, 0 \leqslant q_{2} \leqslant P$ :

- For $\frac{1}{2}<P \leqslant 1$, the line intersects the square in its 'lower corner' or, in other words, the restriction of the line to the first quadrant is entirely inside the square. Then, $\mathcal{P T}_{P}$ is the whole segment between the points $(1-P, 0)$ and $(0,1-P)$ (see figure A1 (left plot)). These are the pure points, and both lead to the same extremal value for $\varphi$. 
- For $\frac{1}{3}<P \leqslant \frac{1}{2}$, the intersection of the line with the square reduces to the segment linking the points $(P, 1-2 P)$ and $(1-2 P, P)$, which are then the pure points of $\mathcal{P T}_{P}$ (see figure A1 (right plot)). Both points lead to the same extremal value for $\varphi$.

Notice that the pure points are on the edges of the square.

A.1.2. Case $N=4$. Now, three different situations arise for the intersection of the plane $q_{1}+q_{2}+q_{3}=1-P$ with the cube $[0 ; P]^{3}$ :

- For $\frac{1}{2}<P \leqslant 1$, the domain $\mathcal{P} \mathcal{T}_{P}$ is the convex body delimited by the triangle of vertices $(1-P, 0,0),(0,1-P, 0)$ and $(0,0,1-P)$ (triangle and its interior); the plane intersects the cube in its 'lower corner' or, in other words, the restriction of the plane to the first octant is entirely inside the cube (see figure A2 (left plot)). The pure points are then all the permutations of $(1-P, 0,0)$, leading all to the same extremal value for $\varphi$. - For $\frac{1}{3}<P \leqslant \frac{1}{2}$, the plane intersects the six facets of the cube, so that $\mathcal{P T} \mathcal{T}_{P}$ is the convex body delimited by the hexagon of vertices $(P, 1-2 P, 0),(1-2 P, P, 0)$, $(0,1-2 P, P),(0, P, 1-2 P),(1-2 P, 0, P),(P, 0,1-2 P)$, which are the pure points (see figure A2 (middle plot)). All of them lead to the same value for $\varphi$.

- For $\frac{1}{4}<P \leqslant \frac{1}{3}$, the plane intersects the cube at its 'higher corner', so that $\mathcal{P T}_{P}$ is the convex body delimited by the triangle of vertices $(P, P, 1-3 P),(1-3 P, P, P)$ and $(P, 1-3 P, P)$, these points being its pure points (see figure A2 (right plot)). Again, these points lead to the same value for $\varphi$.

Notice that the pure points are on the edges of the cube.

\section{A.1.3. Arbitrary $N=$ case: convex polytope $\mathcal{P} \mathcal{T}_{P}$ and minimal $(h, \phi)$-entropy.}

Pure points of the polytope $\mathcal{P T}_{P}$. As previously mentioned, the intersection between a hypercube and a hyperplane is a polytope, convex hull of its vertices that are the pure points of the polytope; moreover, the vertices of the polytope are on edges of the hypercube [87]. Finding the vertices (i.e., the pure points) of such a polytope is not an easy task in general since the number of vertices grows rapidly with dimension $N$ [87]. However, the problem simplifies drastically due to the regularity of the hypercube $\mathcal{H C} \mathcal{C}_{P}=[0 ; P]^{N-1}$. Indeed, the $(N-1) 2^{N-2}$ edges $E_{P}$ are of the form

$$
E_{P}^{\downarrow}=\{(\underbrace{P, \ldots, P}_{M-1 \text { times }}, s, \underbrace{0, \ldots, 0}_{N-M-1 \text { times }}), \quad 0 \leqslant s<P\}
$$

for every $M=1,2, \ldots, N-1$, where $\cdot \downarrow$ denotes the rearrangement of the $(N-1)$-uplet (components put in decreasing order).

A point in $E_{P}$ is a vertex of the polytope $\mathcal{P} \mathcal{T}_{P}$ if it also belongs to $\mathcal{H} \mathcal{P}_{P}$, that is for $s^{*} \in[0 ; P)$ such that $(M-1) P+s^{*}=1-P$, or $M=\frac{1-s^{*}}{P}$, which is greater that $\frac{1}{P}-1$, and less than or equal to $\frac{1}{P}$. Since $M$ is an integer we finally find that, given a value of $P$, the pure points are such that

$$
M=\left\lfloor\frac{1}{P}\right\rfloor \quad \text { and } \quad s^{*}=1-\left\lfloor\frac{1}{P}\right\rfloor P,
$$

where $\lfloor\cdot\rfloor$ denotes the floor part. 

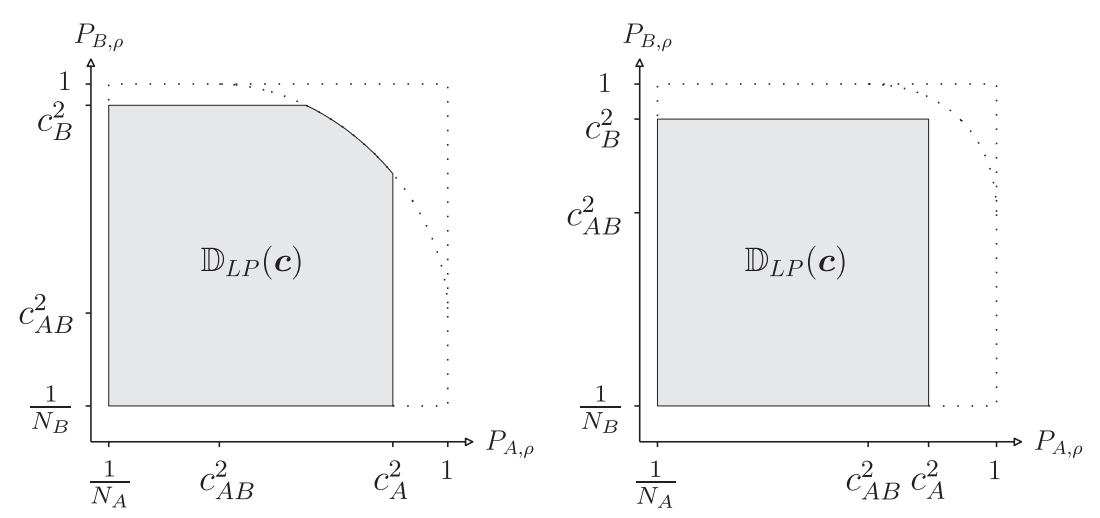

Figure A3. Representation (shaded region) of the domain $\mathbb{D}_{\mathrm{LP}}(\boldsymbol{c})$ (A.10) for pairs of maximal probabilities when $c_{B}^{2}>g_{c_{A, B}}\left(c_{A}^{2}\right)$ (left) and $c_{B}^{2} \leqslant g_{c_{A, B}}\left(c_{A}^{2}\right)$ (right).

This allows us to conclude that the edges of $\mathcal{H C}_{P}$ contain at most one vertex of $\mathcal{P T}_{P}$ (which is intuitive since no facet of $\mathcal{H C} \mathcal{C}_{P}$ is parallel to the hyperplane $\mathcal{H P}_{P}$ ) and that $\mathcal{P} \mathcal{T}_{P}$ is the convex hull of the set of the $(N-M)\left(\begin{array}{c}N-1 \\ M-1\end{array}\right)$ points that belong to $E_{P}$, equation (A.6), for $s$ and $M$ given in equation (A.7). This has been illustrated in the particular cases $N=3$ (with $M=1$ and 2 from left to right in figure A1) and $N=4$ (with $M=1,2$ and 3 from left to right in figure A2).

Optimal vector and minimal entropy. As previously recalled, $\varphi$ being strictly concave (respectively convex), it achieves its minimum (respectively maximum) on the polytope (convex body) $\mathcal{P} \mathcal{T}_{P}$ only in its vertices (pure points). In other words, the minimal entropy solution for the original problem (A.1) is achieved only for the probability vectors of the form

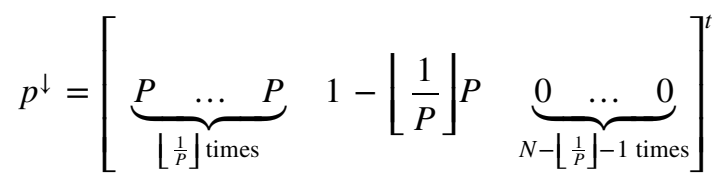

and its expression $H_{(h, \phi)}^{\min }(P) \equiv \min _{p \in \mathcal{P}_{N}: \max _{k} p_{k}=P} H_{(h, \phi)}(p)$ is given by

$$
H_{(h, \phi)}^{\min }(P)=h\left(\left\lfloor\frac{1}{P}\right\rfloor \phi(P)+\phi\left(1-\left\lfloor\frac{1}{P}\right\rfloor P\right)\right),
$$

where $P \in\left[\frac{1}{N} ; 1\right]$

We can verify a posteriori the solution obtained for the minimization problem, using the Schur-concavity of $(h, \phi)$-entropies. Indeed, vector $p$ defined by equation (A.8) majorizes all the probability vectors with maximal probability equal to $P$, and thus its entropy is minimal over these probability vectors.

\section{A.2. Second step: minimization of the sum of minimal $(h, \phi)$-entropies subject to the Landau- Pollak inequality}

Recall that Landau-Pollak inequality links the maximal probabilities $P_{A, \rho}$ and $P_{B, \rho}$ corresponding to the POVMs $A$ and $B$, respectively [82]. We now address the problem of minimization of the sum of minimal $(h, \phi)$-entropies, which is written in terms of $P_{A, \rho}$ and $P_{B, \rho}$, 

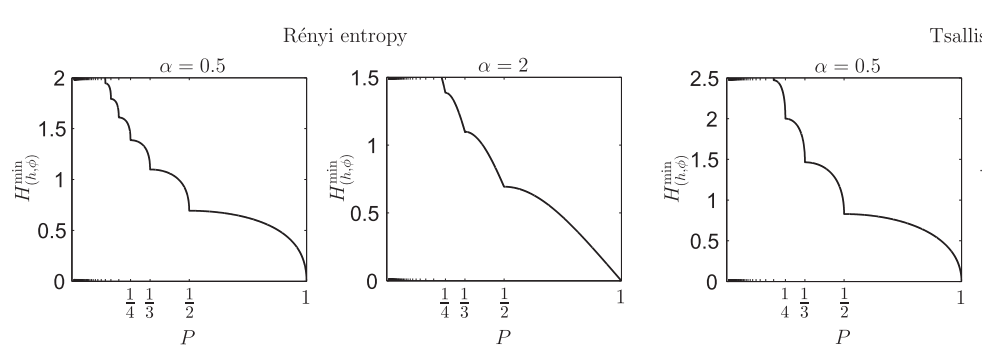

allis entropy

Figure A4. Decreasing behavior of the function $H_{(h, \phi)}^{\min }(P)$ versus $P \in(0 ; 1]$, in the case of Rényi entropy (first and second plots) and in the case of Tsallis entropy (third and fourth plots). Here the entropic index are $\alpha=0.5$ or 2 , as indicated.

under that inequality constraint. We first analyze the domain where the pair $\left(P_{A, \rho}, P_{B, \rho}\right)$ lives and then the behavior of the sum of minimal entropies within this domain. This allows us to slightly simplify the problem.

A.2.1. Representation of the Landau-Pollak inequality domain. Following our previous work [82], it can be seen that the Landau-Pollak inequality constrains the pair of maximal probabilities $\left(P_{A, \rho}, P_{B, \rho}\right)$ in the domain:

$\mathrm{D}_{\mathrm{LP}}(\boldsymbol{c})=\left\{\left(P_{A}, P_{B}\right) \in\left[\frac{1}{N_{A}} ; c_{A}^{2}\right] \times\left[\frac{1}{N_{B}} ; c_{B}^{2}\right]: P_{B} \leqslant g_{c_{A, B}}\left(P_{A}\right)\right.$ when $\left.P_{A} \geqslant c_{A, B}^{2}\right\}$,

where $c=\left(c_{A}, c_{B}, c_{A, B}\right)$ and

$$
g_{c}(x)=\cos ^{2}(\arccos c-\arccos \sqrt{x}) .
$$

If $c_{B}^{2} \leqslant g_{c_{A, B}}\left(c_{A}^{2}\right)$, the allowed domain becomes $\left[\frac{1}{N_{A}} ; c_{A}^{2}\right] \times\left[\frac{1}{N_{B}} ; c_{B}^{2}\right]$. This is represented in figure $\mathrm{A} 3$.

A.2.2. Minimal entropies sum. We have reduced the problem to solve

$$
\min _{\left(P_{A, \rho}, P_{B, \rho}\right) \in \mathrm{D}_{\mathrm{LP}}(\boldsymbol{c})}\left\{H_{\left(h_{A}, \phi_{A}\right)}^{\min }\left(P_{A, \rho}\right)+H_{\left(h_{B}, \phi_{B}\right)}^{\min }\left(P_{B, \rho}\right)\right\}
$$

for given $A, B,\left(h_{A}, \phi_{A}\right)$ and $\left(h_{B}, \phi_{B}\right)$, with $H_{(h, \phi)}^{\min }(P)$ given by equation (A.9). For any $M=1,2, \ldots, N-1$, and for any $P_{1}$ and $P_{2}$ such that $\frac{1}{M+1} \leqslant P_{1} \leqslant P_{2} \leqslant \frac{1}{M}$ we have $\left[\begin{array}{llll}P_{1} \ldots P_{1} & 1-M P_{1} & 0 \ldots & \ldots\end{array}\right]^{t} \prec\left[\begin{array}{llll}P_{2} \ldots P_{2} & 1-M P_{2} & 0 \ldots 0\end{array}\right]^{t}$ and thus, from the Schurconcavity of the $(h, \phi)$-entropy, $H_{(h, \phi)}^{\min }\left(P_{1}\right) \geqslant H_{(h, \phi)}^{\min }\left(P_{2}\right)$. In other words, function $P \mapsto H_{(h, \phi)}^{\min }(P)$ is decreasing in each intervals $\left(\frac{1}{M+1} ; \frac{1}{M}\right)$ and thus, by continuity, in $(0 ; 1]$. This is illustrated in figure A4 in the case of Rényi and Tsallis entropies.

Reasoning by fixing $P_{A, \rho}$ and minimizing the entropies sum over $P_{B, \rho}$ and reversing the roles of $A$ and $B$, we immediately obtain that the minimum is achieved when:

- $\left(P_{A, \rho}, P_{B, \rho}\right)=\left(c_{A}^{2}, c_{B}^{2}\right)$ if $c_{B}^{2} \leqslant g_{c_{A, B}}\left(c_{A}^{2}\right)$. Thus, the minimum takes the analytical form

$$
H_{\left(h_{A}, \phi_{A}\right)}^{\min }\left(c_{A}^{2}\right)+H_{\left(h_{B}, \phi_{B}\right)}^{\min }\left(c_{B}^{2}\right)
$$

or 
- $\left(P_{A, \rho}, P_{B, \rho}\right)$ is in the curve $\left(P_{A, \rho}, g_{c_{A, B}}\left(P_{A, \rho}\right)\right)$ with $P_{A, \rho} \in\left[g_{c_{A, B}}\left(c_{B}^{2}\right), c_{A}^{2}\right]$ if $c_{B}^{2}>g_{c_{A, B}}\left(c_{A}^{2}\right)$.

Let us define the angles

$$
\gamma_{A} \equiv \arccos c_{A}, \quad \gamma_{B} \equiv \arccos c_{B}, \quad \text { and } \quad \gamma_{A, B} \equiv \arccos c_{A, B}
$$

the one-to-one mapping

$$
P_{A, \rho} \equiv \cos ^{2} \theta \quad \text { with } \quad \theta \in\left[\gamma_{A} ; \gamma_{A, B}-\gamma_{B}\right]
$$

leading to

$$
g_{c_{A, B}}\left(P_{A, \rho}\right)=\cos ^{2}\left(\gamma_{A, B}-\theta\right)
$$

with $\gamma_{A, B}-\theta \in\left[\gamma_{A} ; \gamma_{A, B}-\gamma_{B}\right]$, and function

$$
\mathcal{D}_{(h, \phi)}(\theta) \equiv h\left(\left\lfloor\frac{1}{\cos ^{2} \theta}\right\rfloor \phi\left(\cos ^{2} \theta\right)+\phi\left(1-\left\lfloor\frac{1}{\cos ^{2} \theta}\right\rfloor \cos ^{2} \theta\right)\right) .
$$

With these notations,

- condition $c_{B}^{2} \leqslant g_{c_{A, B}}\left(c_{A}^{2}\right)$ simplifies to $\gamma_{B} \geqslant \gamma_{A, B}-\gamma_{A}$,

- $H_{\left(h_{A}, \phi_{A}\right)}\left(c_{A}^{2}\right)=\mathcal{D}_{\left(h_{A}, \phi_{A}\right)}\left(\gamma_{A}\right)$ (and similarly for $B$ ),

- $H_{\left(h_{A}, \phi_{A}\right)}^{\min }\left(P_{A, \rho}\right)=\mathcal{D}_{\left(h_{A}, \phi_{A}\right)}(\theta)$ and $H_{\left(h_{B}, \phi_{B}\right)}^{\min }\left(g_{c_{A, B}}\left(P_{A, \rho}\right)\right)=\mathcal{D}_{\left(h_{B}, \phi_{B}\right)}\left(\gamma_{A, B}-\theta\right)$.

Thus, the minimal entropies sum is given by

$$
\begin{cases}\mathcal{D}_{\left(h_{A}, \phi_{A}\right)}\left(\gamma_{A}\right)+\mathcal{D}_{\left(h_{B}, \phi_{B}\right)}\left(\gamma_{B}\right) & \text { if } \gamma_{A, B} \leqslant \gamma_{A}+\gamma_{B} \\ \min _{\theta \in\left[\gamma_{A}, \gamma_{A, B}-\gamma_{B}\right]}\left(\mathcal{D}_{\left(h_{A}, \phi_{A}\right)}(\theta)+\mathcal{D}_{\left(h_{B}, \phi_{B}\right)}\left(\gamma_{A, B}-\theta\right)\right) & \text { otherwise }\end{cases}
$$

proving the proposition. Note that the cosine being increasing (in the interval $\theta$ lies in), the decreasing property of $H_{(h, \phi)}^{\min }(P)$ implies that $\mathcal{D}_{(h, \phi)}(\theta)$ is increasing versus $\theta$.

\section{Appendix B. Proof of corollary 2}

Remember that in this case, we have $N_{A}=N_{B}=N, c_{A}=c_{B}=1$ and $c_{A, B}=c$.

In [40] we solved the problem in the case of the qubit $(N=2)$ for pure states and for the Rényi entropy. It appears that:

- This result extends for arbitrary pairs of $(h, \phi)$-entropies; the approach [40, appendix A] extends step by step to such entropies, where the concavity (respectively convexity) of $\phi_{B}$ is used instead of the convexity of the mapping $z \mapsto \frac{\Sigma_{k}\left|z_{k}\right|^{\beta}}{\beta-1}$ (see [40, equation (A.13)]) and where the Schur-concavity of $H_{\left(h_{B}, \phi_{B}\right)}$ is used to finish the proof (see [40, equations (A.14)-(A.19) and appendix A.3.2]), which allows to consider functions $h_{B}$ and $\phi_{B}$ nonnecessarily differentiable.

- The extended bound for the qubit and pure states writes precisely under the form equation (22), where $c>\frac{1}{\sqrt{2}}$ and thus $\left\lfloor\frac{1}{\cos ^{2} \theta}\right\rfloor=1$ (the case $c=\frac{1}{\sqrt{2}}$ is recovered by continuity). 
- The minimizing pure states of proposition 2 of [40] expressed through the optimal angles $\theta$ hold, where these angles clearly depend of the pairs of functionals $\left(h_{A}, \phi_{A}\right)$ and $\left(h_{B}, \phi_{B}\right)$.

- Due to the coincidence of bound (22) and the optimal bound for pure states, this bound remains optimal in the mixed states (a pure state being a particular pure state).

Since the case $N=2$ is already treated, let us concentrate on $N \geqslant 3$.

In the context of pure states, one has $\rho=|\Psi\rangle\langle\Psi|$, where $|\Psi\rangle$ is an element of an $N$ dimensional Hilbert space. Using the notation of [40], the state $|\Psi\rangle$ can be expanded on the eigenbases of $A$ and $B$ under the form $|\Psi\rangle=\sum_{i=1}^{N} \psi_{i}\left|a_{i}\right\rangle=\sum_{j=1}^{N} \widetilde{\psi}_{j}\left|b_{j}\right\rangle$. Thus $p_{i}(A, \rho)=\left|\psi_{i}\right|^{2}$ and $p_{j}(B, \rho)=\left|\widetilde{\psi}_{j}\right|^{2}$. Moreover, arranging the complex coefficients $\psi_{i}$ and $\widetilde{\psi}_{j}$ in column vectors, $\psi=\left[\begin{array}{lll}\psi_{1} & \cdots & \psi_{N}\end{array}\right]^{t}$ and $\widetilde{\psi}=\left[\widetilde{\psi}_{1} \cdots \widetilde{\psi}_{N}\right]^{t}$, one can see that these vectors are linked via $\widetilde{\psi}=T \psi$, where $T$ is the transformation matrix whose elements are defined in equation (10).

Now, let us consider $N \times N$ unitary matrices of the form $T=\left[\begin{array}{cc}T^{(2)} & 0 \\ 0 & T^{(N-2)}\end{array}\right]$, where $T^{(n)}$ stands for an $n \times n$ unitary matrix, and we impose the largest-modulus element of $T$ to be 'located' in $T^{(2)}$, that is $c=\max _{i, j}\left|T_{i j}\right|=\max _{i, j}\left|T_{i j}^{(2)}\right|$. This last condition can be fulfilled only if $N \geqslant 4$ because one must have $c \geqslant \max _{i, j}\left|T_{i j}^{(N-2)}\right| \in\left[\frac{1}{\sqrt{N-2}} ; 1\right]$. Let $\left|\Psi^{(2)}\right\rangle=\psi_{1}^{(2)}\left|a_{1}\right\rangle+\psi_{2}^{(2)}\left|a_{2}\right\rangle$ be a minimizing qubit pure state corresponding to the transformation matrix $T^{(2)}$ so that $H_{\left(h_{A}, \phi_{A}\right)}\left(p\left(A, \rho^{(2)}\right)\right)+H_{\left(h_{B}, \phi_{B}\right)}\left(p\left(B, \rho^{(2)}\right)\right)=\widetilde{\mathcal{B}}_{\left(h_{A}, \phi_{A}\right),\left(h_{B}, \phi_{B}\right) ; 2}(c)$ with $\rho^{(2)}=\left|\Psi^{(2)}\right\rangle\left\langle\Psi^{(2)}\right|$. Consider the density operator $\rho=|\Psi\rangle\langle\Psi|$ build up from the extended pure state $|\Psi\rangle$ such that its vector of coefficients is $\psi=\left[\begin{array}{c}\psi^{(2)} \\ 0\end{array}\right]$. Then one has $H_{\left(h_{A}, \phi_{A}\right)}(p(A, \rho))+H_{\left(h_{B}, \phi_{B}\right)}(p(B, \rho))=H_{\left(h_{A}, \phi_{A}\right)}\left(p\left(A, \rho^{(2)}\right)\right)+H_{\left(h_{B}, \phi_{B}\right)}\left(p\left(B, \rho^{(2)}\right)\right)=$ $\widetilde{\mathcal{B}}_{\left(h_{A}, \phi_{A}\right),\left(h_{B}, \phi_{B}\right) ; 2}(c)=\mathcal{B}_{\left(h_{A}, \phi_{A}\right),\left(h_{B}, \phi_{B}\right)}(c)$. The last equality comes from the coincidence between the $c$-optimal bound for the qubit case (see above), and expression (22). Finally, by definition of $c$-optimal bound, one has both $\mathcal{B}_{\left(h_{A}, \phi_{A}\right),\left(h_{B}, \phi_{B}\right)}(c) \leqslant \widetilde{\mathcal{B}}_{\left(h_{A}, \phi_{A}\right),\left(h_{B}, \phi_{B}\right) ; N}(c)$ and $\mathcal{B}_{\left(h_{A}, \phi_{A}\right),\left(h_{B}, \phi_{B}\right)}(c)=H_{\left(h_{A}, \phi_{A}\right)}(p(A, \rho))+H_{\left(h_{B}, \phi_{B}\right)}(p(B, \rho)) \geqslant \widetilde{\mathcal{B}}_{\left(h_{A}, \phi_{A}\right),\left(h_{B}, \phi_{B}\right) ; N}(c)$, proving the $c$-optimality of (22) when $c>\frac{1}{\sqrt{2}}$ and $N \geqslant 4$.

The problem of the $c$-optimality of the bound for $N=3$ remains open. We suspect that it is so but we have not been able to prove this yet.

\section{Appendix C. Proof of corollary 3}

It can be seen that our bound (22) in the case of Rényi entropy when $\alpha$ and $\beta$ are sufficiently large, gives $\mathcal{B}_{\infty, \infty} ; \log (c)=\min _{\theta \in[0 ; \gamma]}[-2 \log (\cos \theta)-2 \log (\cos (\gamma-\theta))]$. The minimum is attained for $\theta=\frac{\gamma}{2}$ so that we recover the Deutsch bound: $\mathcal{B}_{\infty, \infty} ; \log (c)=$ $-2 \log \left(\frac{1+c}{2}\right)=\mathcal{B}^{D}(c)$. Now, consider our bound $\mathcal{B}_{\alpha, \beta} ; \log (c)$ which is the solution of the minimization (22), and the probability $P_{A}$ for which the minimum is attained. Since Rényi entropy decreases versus the entropic index, we have $\mathcal{B}_{\alpha, \beta ; \log }(c)=R_{\alpha}^{\min }\left(P_{A}\right)+$ $R_{\beta}^{\min }\left(g_{c}\left(P_{A}\right)\right) \geqslant R_{\infty}^{\min }\left(P_{A}\right)+R_{\infty}^{\min }\left(g_{c}\left(P_{A}\right)\right) \geqslant \mathcal{B}_{\infty, \infty} ; \log (c)=\mathcal{B}^{D}(c)$, where $R_{\lambda}^{\min } \equiv$ $H_{\left(\frac{\log }{1-\lambda}, \mathrm{id}^{\lambda}\right)}$, that proves that our bound improves Deutsch one. 


\section{Appendix D. Proof of corollary 4}

Let us consider the extreme pair of indices $(\alpha, \beta)=(0,0)$, and go back to expression (22) for the bound

$$
\mathcal{B}_{0,0 ; f}(c)=\min _{P_{A, \rho} \in\left[c^{2} ; 1\right]}\left[R_{0}^{\min }\left(P_{A, \rho}\right)+R_{0}^{\min }\left(g_{c}\left(P_{A, \rho}\right)\right)\right] .
$$

By symmetry of the quantity in square brackets, since $g_{c}\left(\frac{1+c}{2}\right)=\frac{1+c}{2}$, one can restrict the search for $P_{A, \rho}$ to the interval $\left[c^{2} ; \frac{1+c}{2}\right]$. Then:

- For $P_{A, \rho}=c^{2} \quad$ one has $g_{c}\left(P_{A, \rho}\right)=1 \quad$ and thus $R_{0}^{\min }\left(g_{c}\left(P_{A, \rho}\right)\right)=0 \quad$ while $R_{0}^{\min }\left(P_{A, \rho}\right)=\log \left(\left\lceil\frac{1}{c^{2}}\right\rceil\right)$.

- For $P_{A, \rho} \in\left(c^{2} ; \frac{1+c}{2}\right]$ one has $g_{c}\left(P_{A, \rho}\right) \in\left[\frac{1+c}{2} ; 1\right) \subset\left(\frac{1}{2} ; 1\right)$ and thus $R_{0}^{\min }\left(g_{c}\left(P_{A, \rho}\right)\right)=\log 2$. A rapid inspection of $R_{0}^{\min }\left(P_{A, \rho}\right)$ allows one to prove that in this interval it decreases versus $P_{A, \rho}$ and that the minimum is also $\log 2$. Thus

$$
\min _{P_{A, \rho} \in\left(c^{2} ; \frac{1+c}{2}\right]}\left[R_{0}^{\min }\left(P_{A, \rho}\right)+R_{0}^{\min }\left(g_{c}\left(P_{A, \rho}\right)\right)\right]=2 \log 2 .
$$

Therefore

$$
\mathcal{B}_{0,0 ; \log }(c)=\min \left\{2 \log 2, \log \left(\left[\frac{1}{c^{2}}\right]\right)\right\} .
$$

Now, when $c \leqslant \frac{1}{2}$, we have $\mathcal{B}^{\mathrm{MU}}(c)=-2 \log c \geqslant \log 4=\mathcal{B}_{0,0 ;} \log (c)$. Moreover, in this case $\mathcal{B}_{0,0 ; \log }(c)=2 R_{0}^{\min }\left(\frac{1+c}{2}\right)$ so that by using the decreasing property of $R_{\lambda}^{\min }$ versus $\lambda$ we obtain

$$
\begin{aligned}
\mathcal{B}_{\alpha, \beta ; \log }(c) & \leqslant R_{\alpha}^{\min }\left(\frac{1+c}{2}\right)+R_{\beta}^{\min }\left(\frac{1+c}{2}\right) \leqslant 2 R_{0}^{\min }\left(\frac{1+c}{2}\right) \\
& =\mathcal{B}_{0,0 ; \log }(c) \leqslant \mathcal{B}^{\mathrm{MU}}(c)
\end{aligned}
$$

that concludes the proof.

\section{References}

[1] Heisenberg W 1927 Über den anschaulichen inhalt der quantentheoretischen kinematik und mechanik Z. Phys. 43 172-98

[2] Kennard E H 1927 Zur quantenmechanik einfacher bewegungstypen Z. Phys. 44 326-52

[3] Robertson H P 1929 The uncertainty principle Phys. Rev. 34 163-4

[4] Samorodnitsky G and Taqqu M S 1994 Stable Non-Gaussian Random Processes. Stochastic Models with Infinite Variance (New York: Chapman and Hall)

[5] Deutsch D 1983 Uncertainty in quantum measurements Phys. Rev. Lett. 50 631-3

[6] Maassen H and Uffink J B M 1988 Generalized entropic uncertainty relations Phys. Rev. Lett. 60 1103-6

[7] Luis A 2001 Complementary and certainty relations for two-dimensional systems Phys. Rev. A 64 012103

[8] Luis A 2011 Effect of fluctuations measures on the uncertainty relations between two observables: different measures lead to opposite conclusions Phys. Rev. A 84034101 
[9] Zozor S 2012 Bruit, non-linéaire et information: quelques résultats Habilitation à Diriger des Recherches (Grenoble: Institut National Polytechnique de Grenoble)

[10] Shannon C E 1948 A mathematical theory of communication Bell Syst. Tech. J. 27 623-56

[11] Rényi A 1961 On measures of entropy and information Proc. 4th Berkeley Symp. on Mathematical Statistics and Probability 1 547-61

[12] Cover T M and Thomas J A 2006 Elements of Information Theory 2nd edn (Hoboken, NJ: Wiley)

[13] Hirschman I I 1957 A note on entropy Am. J. Math. 79 152-6

[14] Białynicki-Birula I and Mycielski J 1975 Uncertainty relations for information entropy in wave mechanics Commun. Math. Phys. 44 129-32

[15] Beckner W 1975 Inequalities in Fourier analysis Ann. Math. 102 159-82

[16] Kraus K 1987 Complementary observables and uncertainty relations Phys. Rev. D 35 3070-5

[17] Białynicki-Birula I 1984 Entropic uncertainty relations Phys. Lett. A 103 253-4

[18] Rajagopal A K 1995 The Sobolev inequality and the Tsallis entropic uncertainty relation Phys. Lett. A 205 32-36

[19] Sánchez-Ruiz J 1995 Improved bounds in the entropic uncertainty and certainty relations for complementary observables Phys. Lett. A 201 125-31

[20] Portesi M and Plastino A 1996 Generalized entropy as measure of quantum uncertainty Physica A 225 412-30

[21] Sánchez-Ruiz J 1998 Optimal entropic uncertainty relation in two-dimensional Hilbert space Phys. Lett. A 244 189-95

[22] Ghirardi G C, Marinatto L and Romano R 2003 An optimal entropic uncertainty relation in a twodimensional Hilbert space Phys. Lett. A 317 32-36

[23] Biaynicki-Birula I 2006 Formulation of the uncertainty relations in terms of the Rényi entropies Phys. Rev. A $\mathbf{7 4} 052101$

[24] Zozor S and Vignat C 2007 On classes of non-Gaussian asymptotic minimizers in entropic uncertainty principles Physica A 375 499-517

[25] Luis A 2007 Quantum properties of exponential states Phys. Rev. A 75052115

[26] de Vicente J I and Sánchez-Ruiz J 2008 Improved bounds on entropic uncertainty relations Phys. Rev. A 77042110

[27] Zozor S, Portesi M and Vignat C 2008 Some extensions to the uncertainty principle Physica A 387 4800-8

[28] Wu S, Yu S and Mølmer K 2009 Entropic uncertainty relation for mutually unbiased bases Phys. Rev. A 79022104

[29] Wehner S and Winter A 2010 Entropic uncertainty relations-a survey New J. Phys. 12025009

[30] Bialynicki-Birula I and Rudnicki $Ł 2010$ Entropic uncertainty relations in quantum physics Statistical Complexity. Application in Electronic Structure ed K D Sen (Berlin: Springer) chapter 1

[31] Dehesa J S, López-Rosa S and Manzano D 2010 Entropy and complexity analyses of Ddimensional quantum systems Statistical Complexities: Application to Electronic Structure ed K D Sen (Berlin: Springer) chapter 5

[32] Tomamichel M and Renner R 2011 Uncertainty relation for smooth entropies Phys. Rev. Lett. 106 110506

[33] Coles P J, Yu L, Gheorghiu V and Griffiths R B 2011 Information-theoretic treatment of tripartite systems and quantum channels Phys. Rev. A 83062338

[34] Bosyk G M, Portesi M, Plastino A and Zozor S 2011 Comment on 'improved bounds on entropic uncertainty relations' Phys. Rev. A 84056101

[35] Rastegin A E 2011 Entropic uncertainty relations for extremal unravelings super-operators J. Phys. A: Math. Theor. 44095303

[36] Rastegin A E 2011 Entropic formulation of the uncertainty principle for the number and annihilation operators Phys. Scr. 84057001

[37] Rastegin A E 2012 Notes on entropic uncertainty relations beyond the scope of Riesz' therorem Int. J. Theor. Phys. 51 1300-14

[38] Coles P J, Colbeck R, Yu L and Zwolak M 2012 Uncertainty relations from simple entropic properties Phys. Rev. Lett. 108210405

[39] Bosyk G M, Portesi M and Plastino A 2012 Collision entropy and optimal uncertainty Phys. Rev. A 65012108

[40] Zozor S, Bosyk G M and Portesi M 2013 On a generalized entropic uncertainty relation in the case of the qubit J. Phys. A: Math. Theor. 46465301 
[41] Puchała Z, Rudnicki $Ł$ and Życzkowski K 2013 Entropic uncertainty relations and majorization J. Phys. A: Math. Theor. 46272002

[42] Friedland S, Gheorghiu V and Gour G 2013 Universal uncertainty relations Phys. Rev. Lett. 111 230401

[43] Bosyk G M, Portesi M, Holik F and Plastino A 2013 On the connection between complementary and uncertainty principle in the Mach-Zehnder interferometric setting Phys. Scr. 87065002

[44] Coles P J and Piani M 2014 Improved entropic uncertainty relations and information exclusion relations Phys. Rev. A 89022112

[45] Rudnicki Ł, Puchała Z and Życzkowski K 2014 Strong majorization entropic uncertainty relations Phys. Rev. A 89052115

[46] Huang Y 2012 Variance-based uncertainty relations Phys. Rev. A 86024101

[47] Romera E, Angulo J C and Dehesa J S 1999 Fisher entropy and uncertainty like relationships in many-body systems Phys. Rev. A 59 4064-7

[48] Romera E, Sánchez-Moreno P and Dehesa J S 2006 Uncertainty relation for Fisher information of $D$-dimensional single-particle systems with central potentials J. Math. Phys. 47103504

[49] Sánchez-Moreno P, González-Férez R and Dehesa J S 2006 Improvement of the Heisenberg and Fisher-information-based uncertainty relations for D-dimensional potentials New J. Phys. 8330

[50] Zozor S, Portesi M, Sánchez-Moreno P and Dehesa J S 2011 Position-momentum uncertainty relation based on moments of arbitrary order Phys. Rev. A 83052107

[51] Salicrú M, Menéndez M L, Morales D and Pardo L 1993 Asymptotic distribution of $(h, \phi)$ entropies Commun. Stat.-Theory Methods 22 2015-31

[52] Menéndez M L, Morales D, Pardo L and Salicrú M $1997(h, \phi)$-entropy differential metric Appl. Math. 42 81-98

[53] Landau H J and Pollak H O 1961 Prolate spheroidal wave functions, Fourier analysis and uncertainty: II Bell Syst. Tech. J. 40 65-84

[54] Havrda J and Charvát F 1967 Quantification method of classification processes: concept of structural $\alpha$-entropy Kybernetika 3 30-35

[55] Daróczy Z 1970 Generalized information functions Inf. Control 16 36-51

[56] Tsallis C 1988 Possible generalization of Boltzmann-Gibbs statistics J. Stat. Phys. 52 479-87

[57] Ricaud B and Torrésani B 2013 Refined support and entropic uncertainty inequalities IEEE Trans. Inf. Theor. 59 4272-9

[58] Elad M and Bruckstein A M 2002 A generalized uncertainty principle and sparse representation in pairs of bases IEEE Trans. Inf. Theor. 48 2558-67

[59] Ghobber S and Jaming P 2011 On uncertainty principles in the finite dimensional setting Linear Algebr. Appl. 435 751-68

[60] Karamata J 1932 Sur une inegalité relative aux fonctions convexes Publ. Math. Univ. Belgrade 1 $145-8$

[61] Marshall A W, Olkin I and Arnold B C 2011 Inequalities: Theory of Majorization and Its Applications 2nd edn (New York: Springer)

[62] Cambini A and Martein L 2009 Generalized Convexity and Optimization: Theory and Applications (Heidelberg: Springer)

[63] Andréasson N, Evgrafov A and Patriksson M 2007 An Introduction to Continuous Optimization (Lund: Studentlitteratur AB)

[64] Bengtsson I and Życzkowski K 2006 Geometry of Quantum States: an Introduction to Quantum Entanglement (Cambridge: Cambridge University Press)

[65] Hardy G, Littlewood J E and Pólya G 1952 Inequalities 2nd edn (Cambridge: Cambridge University Press)

[66] Rastegin A E 2010 Rényi formulation of the entropic uncertainty principle for POVMs J. Phys. A: Math. Theor. 43155302

[67] Berta M, Christandl M, Colbeck R, Renes J M and Renner R 2010 The uncertainty principle in the presence of quantum memory Nat. Phys. 6 659-62

[68] Müller-Lennert M, Dupuis F, Szehr O and Fehr S 2013 On quantum Rényi entropies: a new generalization and some properties J. Math. Phys. 54122203

[69] Frank R and Lieb E H 2013 Extended quantum conditional entropy and quantum uncertainty inequalities Commun. Math. Phys. 323 487-95

[70] Ivanovic I D 1992 An inequality for the sum of entropies of unbiased quantum measurements J. Phys. A: Math. Gen. 25 363-4 
[71] Ballester M and Wehner S 2007 Entropic uncertainty relations and locking: tight bounds for mutually unbiased bases Phys. Rev. A 75022319

[72] Huang Y 2011 Entropic uncertainty relations in multidimensional position and momentum spaces Phys. Rev. A 83052124

[73] Frank R and Lieb E H 2012 Entropy and the uncertainty principles Ann. Henri Poincaré 13 $1711-7$

[74] Berta M, Christandl M, Furrer F, Scholz V B and Tomamichel M 2013 Continuous variable entropic uncertainty relations in the presence of quantum memory arXiv:1308.4527

[75] Giovannetti V 2004 Separability conditions from entropic uncertainty relations Phys. Rev. A 70 012102

[76] Gühne O and Lewenstein M 2004 On entropic uncertainty relations and entanglement Phys. Rev. A 70022316

[77] Huang Y 2010 Entanglement criteria via concave-function uncertainty relations Phys. Rev. A 82 012335

[78] Ng N H Y, Berta M and Wehner S 2012 Min-entropy uncertainty relation for finite-size cryptography Phys. Rev. A 86042315

[79] Furrer F, Franz T, Berta M, Leverrier A, Scholz V B, Tomamichel M and Werner R F 2012 Continuous variable quantum key distribution: finite-key analysis of composable security against coherent attacks Phys. Rev. Lett. 109100502

[80] Schneeloch J, Broadbent C J and Howell J C 2014 Improving Einstein-Podolsky-Rosen steering inequalities with state information Phys. Lett. A 378 766-9

[81] Bosyk G M, Osán T M, Lamberti P W and Portesi M 2014 Geometric formulation of the uncertainty principle Phys. Rev. A 89034101

[82] Bosyk G M, Zozor S, Portesi M, Osán T M and Lamberti P W 2014 Geometric approach to extend Landau-Pollak uncertainty relations for positive operator-valued measures Phys. Rev. A 90 052114

[83] Życzkowski K and Kuś M 1994 Random unitary matrices J. Phys. A: Math. Gen. 27 4235-45

[84] Mezzadri F 2007 How to generate random matrices from the classical compact groups Not. Am. Math. Soc. 54 592-604

[85] Feder M and Merhav N 1994 Relations between entropy and error probability IEEE Trans. Inf. Theory 40 259-66

[86] Miller R E 2000 Optimization: Foundations and Applications (New York: Wiley)

[87] Lara C, Flores J J and Calderon F 2009 On the hyperbox-hyperplane intersection problem INFOCOMP: J. Comput. Sci. 8 21-27

[88] Eggleston H G 1958 Convexity (London: Cambridge University Press)

[89] Berger M 1987 Geometry II (Heidelberg: Springer)

[90] Roberts A W and Varberg D E 1973 Convex Functions (New York: Academic) 